\title{
Urbanización de viviendas y gestión ecoeficiente de residuos de construcción en Chile: aplicación del modelo español
}

\author{
Urbanisation of housing and eco-efficient management of \\ construction waste in Chile: application of the Spanish \\ model
}

\begin{abstract}
Claudia Marcela Muñoz Sanguinetti
Cristina Rivero Camacho

Madelyn Marrero Meléndez

Gabriel Cereceda Balic

Resumen

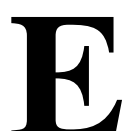

ste trabajo puso en relieve una experiencia pionera en Chile en lo que se refiere a la gestión de los residuos de construcción y demolición (RCD). El caso estudiado corresponde a la construcción de 17 viviendas unifamiliares ubicadas en un condominio habitacional en la ciudad de Temuco, Región de la Araucanía. Para su implementación se aplicó una metodología basada en un modelo de gestión de residuos desarrollado en España para estimar los RCD. Con la adopción de esta metodología de separación en obra de los residuos, los costos materiales se redujeron a la mitad; también se redujo el costo medioambiental de las obras en construcción. Con ello se ha comprobado que es viable la adaptación de la metodología española a un proyecto de urbanización chileno. Además, la aplicación de esta metodología genera un triple beneficio que repercute en lo ambiental, lo social y lo económico. Al mismo tiempo, se ha comprobado que la reutilización, reciclaje, tratamiento o eliminación de los residuos genera nuevos desafíos en el plano legislativo y de gestión de gobierno, elementos a considerar si Chile aspira a alcanzar estándares de países como Brasil o los de la Comunidad Europea en lo que respecta a la gestión de RCD.

Palabras-clave: Residuos de construcción y demolición. Costos de residuos. Cuantificación de residuos. Ecoeficiencia. Urbanización.

\section{${ }^{1}$ Claudia Marcela Muñoz ${ }^{1}$ Universidad del Bío-Bío Concepción - Chile} Sanguinetti

${ }^{2}$ Cristina Rivero Camacho ${ }^{2}$ Universidad de Sevilla Sevilla - España

${ }^{3}$ Madelyn Marrero Meléndez ${ }^{3}$ Universidad de Sevilla Sevilla - España

${ }^{4}$ Gabriel Cereceda Balic 4Universidad del Bío-Bío Concepción - Chile

Recebido em 20/11/18 Aceito em 29/01/19

\section{Abstract}

This paper summarises a pioneering experience in construction and demolition waste management (C\&DW) in Chile. The case study consists of the construction of 17 detached dwellings that are part of an urbanisation project in the city of Temuco, in the Araucana region. The methodology developed in Spain for the C\&DW quantification is employed for the calculation of expected waste in construction projects. The implementation of the proposed methodology, which includes waste separation activities on the construction site, can halve management costs, as well as the environmental cost of construction works. The case study shows the feasibility and adaptation of the Spanish methodology to an urbanisation project in Chile. Furthermore, its application generates triple benefits: environmental, social and economic. At the same time, the new proposal to reuse, recycle, treat or eliminate the waste produced sets new challenges in the areas of legislation and government management, which are elements to be considered if Chile is to achieve $C \& D W$ managemant standards similar to those in place in Brazil and the European Union.

Keywords: Construction and Demolition Waste. Waste costs. Quantification of waste. Ecoefficiency. Urbanization.
\end{abstract}

SANGUINETTI, C. M. M.; CAMACHO, C. R.; MELÉNDEZ, M. M.; BALIC, G. C. Urbanización de viviendas y gestión ecoeficiente de residuos de construcción en Chile: aplicación del modelo español. Ambiente Construído,

Porto Alegre, v. 19, n. 3, p. 275-294, jul./ set. 2019.

ISSN 1678-8621 Associação Nacional de Tecnologia do Ambiente Construído.

http:// dx. doi. org/ 10. 1590/ s1678-86212019000300338 


\section{Introducción}

En esta introducción se examinan los conceptos fundamentales, los antecedentes que competen a la gestión de RCD, su situación en el mundo y en Chile, la metodología más relevante y los modelos de cuantificación.

\section{Concepto de ciclo de vida}

Todos los procesos productivos originan residuos, siendo la construcción una de las industrias con mayor generación. Este tipo de residuos reciben internacionalmente el nombre RCD (MACIEL; STUMPF; KERN, 2016) y originan diversas problemáticas, como la contaminación de los suelos y acuíferos por los vertidos incontrolados, el deterioro del paisaje y la eliminación sin reciclado o reutilización (MORÁN DEL POZO et al., 2011). A nivel mundial son conocidas las problemáticas que involucran la correcta gestión de los RCD y, en particular, su gestión de fin de vida (YUAN et al., 2011; WANG et al., 2010; KOFOWOROLA; GHEEWALA, 2009).

Por lo anterior, es de gran relevancia incorporar el concepto de ciclo de vida de los materiales de construcción para fomentar, en primera instancia, la recuperación de materia prima en las plantas de tratamiento y, en segunda instancia, minimizar los impactos generados al eliminar los RCD que no se pueden aprovechar. Además de involucrar a las empresas constructoras, dicho concepto puede ayudar a potenciar a todas las empresas que suministran materiales al sector construcción, creciendo en su gestión con una filosofía de cuidado del medio ambiente (BELTRÁN RIAÑO, 2017).

\section{Gestion de RCD en el mundo}

Los países más desarrollados en la gestión de residuos alcanzan tasas de recuperación muy altas, siendo de $100 \%$ en Malta, $100 \%$ en Holanda, $96 \%$ en Reino Unido, 94\% en Alemania y 90\% en Dinamarca (EUROSTAT, 2018). Por ejemplo, en 2007, la ciudad de San Francisco (California, Estados Unidos) obligó a las empresas del sector de la construcción a utilizar material reciclado en al menos 2/3 de las necesidades totales, aplicando el conocido principio de "quien contamina, paga", imponiendo sanciones a los no cumplidores (HORRACH, 2017).
En Latinoamérica, Brasil ha sido el primer país a establecer políticas para la correcta gestión de los RCD, obligando a los constructores a dar un mejor manejo a los residuos generados e incentivar la clasificación de éstos en obra. En Brasil, la cantidad de RCD varía entre 230 y 760 kg/habitante/año, dependiendo de la ciudad estudiada; también se estima que en algunas localidades dichos volúmenes lleguen a ocupar el 50\% del vertido total. En el sur de Brasil se catastraron 20 obras que generaron un promedio de $0,128 \mathrm{~m}^{3} / \mathrm{m}^{2}$ a 0,162 $\mathrm{m}^{3} / \mathrm{m}^{2}$ construido (MACIEL; STUMPF; KERN, 2016). A la vez, se han fomentado elementos estratégicos de gestión en las constructoras, pero no se han mostrado efectivos aún (SOUSA; CÂNDIDO; BARROS, 2018). También se han introducido mejoras en el control y la eliminación de los vertidos para prevenir la degradación del entorno (MOCHE SCHNEIDER; PHILIPPI JUNIOR, 2004).

En otros países latinoamericanos, como Colombia, México y Argentina, la gestión y el manejo de los RCD ya están ordenados. Sin embargo, las normas no son cumplidas por parte de muchos de los agentes relacionados, perjudicando el entorno y aumentando la cantidad de escombreras ilegales (SUÁREZ-SILGADO et al., 2018).

En Europa, la Directiva 2008/98/CE establece los conceptos básicos y las definiciones relacionados con la gestión de residuos e incluye dos objetivos de reciclado y recuperación para 2020: el primero corresponde al $50 \%$ de preparación para reutilización y reciclaje de ciertos materiales de desecho de los hogares y otros orígenes similares a los hogares; el segundo se refiere al 70\% de preparación para reutilización, reciclaje y otra recuperación de los RCD. La Directiva exige que los Estados miembros adopten planes de gestión de residuos y programas de prevención para poder revertir el panorama actual y alcanzar un sistema más sostenible (Figura 1).

\section{España y el modelo de alcores}

En España, la fase de obtención y producción de materiales y la de demolición es la que más RCD genera (Figura 2 (DEL RIO; VILLORIA; TORRIJOS, 2017)). 
Figura 1 - J erarquía europea de la gestión de residuos

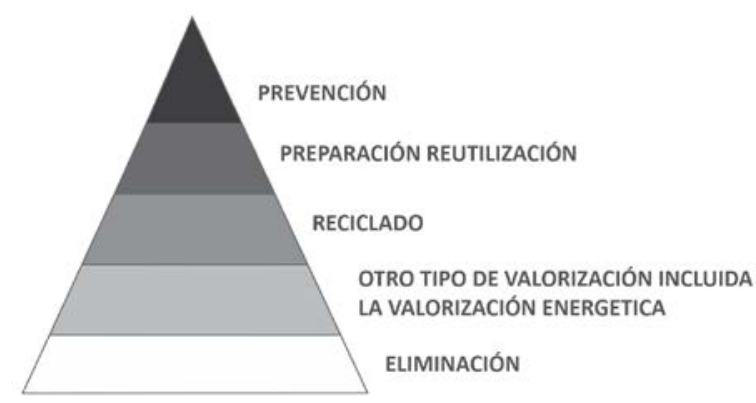

SITUACIÓN ACTUAL

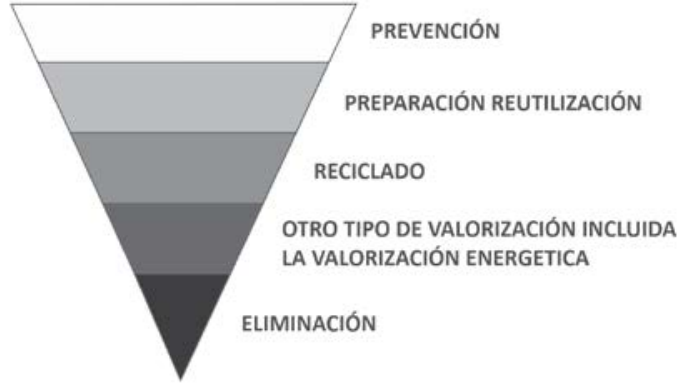

OBJETIVO 2020

Fuente: Unión Europea (2008).

\section{Figura 2 - Residuos generados a lo largo del ciclo de vida de los edificios}

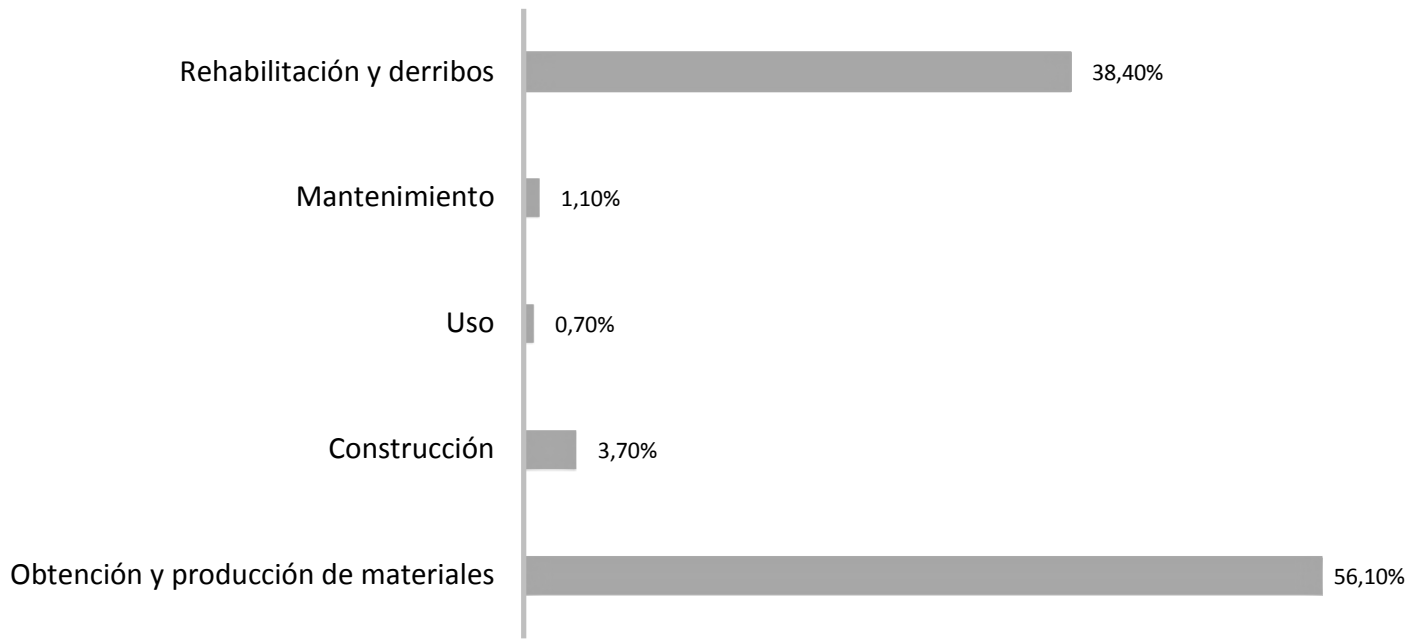

Fuente: elaboración propia a partir de Del Rio, Villoria y Torrijos (2017).

A partir de 2008 se generó un reglamento jurídico sobre la gestión y producción de RCD denominado Real Decreto 105/2008 (ESPAÑA, 2008), que busca ordenar y jerarquizar los residuos, fomentando su prevención, reutilización y reciclaje, valorizando algunos residuos y procurando una adecuada eliminación y tratamiento. Este reglamento permitió un desarrollo sostenible de la actividad de construcción y, después de un periodo de adaptación, se hizo obligatoria para todos los RCD generados en el país (ESPAÑA, 2008). Con la finalidad de minimizar la cantidad de residuos en España, la Ley 22/2011 modificada el 12 de mayo de 2016 aplica el principio de "quien contamina, paga”; esta ley también incluye un artículo relativo a los costos de gestión de residuos, los cuales recaen sobre su productor (JEFATURA..., 2016).

El modelo de gestión de residuos español se prueba por primera vez en la Mancomunidad de los Alcores, por lo que recibe el nombre de "modelo
Alcores”. Este modelo define un sistema de bucle cerrado que fija una fianza que asegure que el promotor del proyecto gestione de forma adecuada los residuos. Al promotor se lo define como “productor de RCD” o “cualquier persona física o jurídica propietaria del inmueble, estructura o infraestructura que lo origina”. Este modelo es implementado por medio de un software empleado en las municipalidades de la Provincia de Sevilla, España (MARRERO et al., 2011). El modelo Alcores se puede resumir en los siguientes 6 pasos:

(a) solicitud de licencia de obras;

(b) informe de evaluación de RCD;

(c) correcta gestión de RCD;

(d) reciclaje;

(e) emisión del certificado de correcta gestión; y

(f) devolución de la fianza. 
La mejora ambiental más importante obtenida a partir de este modelo es la prevención de vertidos ilegales y el reciclado de los RCD, logrando proyectos de construcción más ecoeficientes. Un aspecto relevante del modelo de Alcores es la posibilidad de revalorizar los residuos a partir de un concesionario. Esto implica considerar dos pasos previos: en primer lugar, la caracterización fisicoquímica de los RCD; en segundo lugar, las posibilidades de reciclado (RAMÍREZ-DEARELLANO et al., 2002). Estas etapas previas son las que se deben incorporar en la gestión de RCD en Chile, permitiendo generar un control de las magnitudes esperadas y de los RCD que llegan a vertederos autorizados o plantas de reciclaje, dando prioridad a la minimización de RCD, ya que, en todos los países investigados y los respectivos estudios analizados, alrededor del $40 \%$ de los residuos son de tipo inerte, lo cual facilitaría su reducción y reciclaje.

A continuación, la Figura 3 presenta el modelo Alcores.

\section{Situación de RCD en Chile}

El sector de construcción chileno es una de las grandes industrias generadoras de fuentes de trabajo y una de las actividades industriales con mayor demanda, según datos del informe de macroeconomía y construcción de la Cámara Chilena de la Construcción. La construcción en altura con departamentos continúa aumentando en la industria de construcción residencial, concentrando el $68 \%$ de la superficie autorizada para viviendas a febrero de 2018. En comparación a seis años atrás, los departamentos han casi duplicado su importancia relativa en el sector vivienda (CÁMARA..., 2018).

\section{Marco legislativo}

En Chile el marco normativo asociado a la gestión de residuos surge en 1967 con la publicación del Código Sanitario en el diario oficial. Dicho código regula aspectos específicos asociados a higiene y seguridad del ambiente y de los lugares de trabajo (SERRA NAVARRETE, 2014). Pero el conocimiento del Código Sanitario es limitado. Estudios de la Corporación de Desarrollo Tecnológico (CDT) indican que $60 \%$ de las empresas de construcción no conoce las normativas asociadas a la gestión de residuos (MORAGA, 2018).

\section{Figura 3 - Modelo Alcores de gestión de RCD}

Fuente: Marrero et al. (2011).

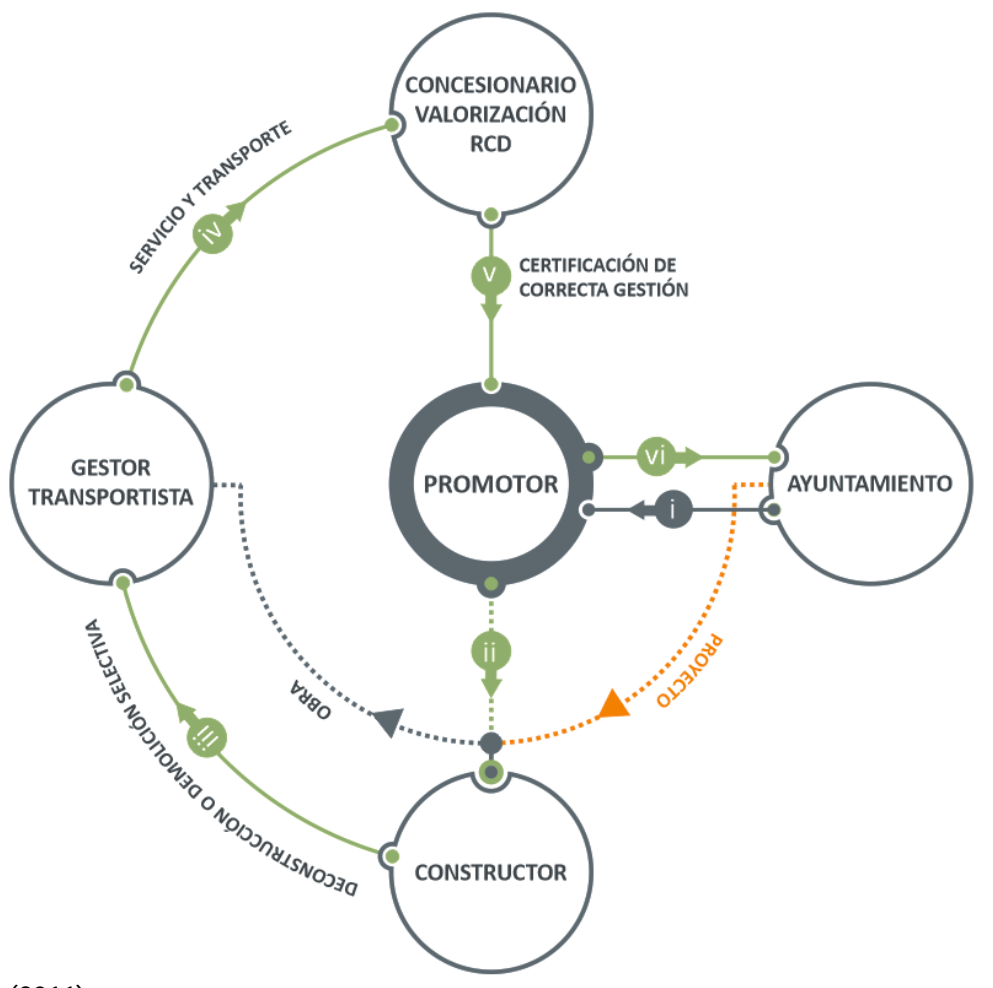


Recientemente se cumplieron dos años de la promulgación de la primera ley de fomento al reciclaje de Chile, la Ley 20.920 (INSTITUTO..., 2016), que establece el marco para la gestión de residuos, la responsabilidad extendida al productor y fomento al reciclaje, una iniciativa pionera en Latinoamérica. Esta ley es el inicio de la institucionalización de la economía circular, que privilegia el reciclaje de materias primas y la valorización energética de residuos antes que optar por la obligación de disponer estos "residuos” en rellenos sanitarios. Por medio de dicha ley, se creó también la denominada responsabilidad extendida al productor como instrumento regulatorio fundamental que busca que los importadores o fabricantes de determinados bienes, una vez terminada su vida útil, se hagan cargo de su recolección y valorización bajo la responsabilidad de un regulador. Lo anterior, le permite al país ahorrar en energía y en uso de materias primas vírgenes, disminuyendo la presión de explotación de recursos naturales.

En cuanto al reciclaje en Chile, luego de dos años y cuatro meses de publicarse la Ley 20.920 (INSTITUTO..., 2016) para la gestión de residuos, la responsabilidad extendida al productor y fomento al reciclaje, ya está listo el primer reglamento que define las metas de valorización y recolección de uno de sus seis productos prioritarios, los neumáticos, esperando en el corto plazo incorporar los reglamentos restantes. Las metas de recolección para sus productores en Chile (fabricantes o importadores) debe ser de 50\% hasta 2021, aumentando hasta $90 \%$ en 2028. Igualmente, las metas de valorización comienzan en 25\% en 2021 y aumentan con el tiempo. Para cumplir dichas metas, los productores tendrán la obligación de organizar y financiar los llamados sistemas de gestión, cuyo objetivo es la recuperación de residuos de todos los sectores productivos (INSTITUTO..., 2016).

\section{Programa Construye 2025}

Otra iniciativa chilena es el Programa Construye 2025 (BRITO, 2018), que es un programa de acción público-privado que busca activar la sustentabilidad en el sector de construcción, considerando todas las etapas del ciclo de vida de un proyecto, desde el suministro de materiales hasta el final de la vida útil de la edificación. Dicho programa promueve una mayor eficiencia en los procesos, desarrollo tecnológico, industrialización, estandarización, plataformas de gestión de proyectos, fortalecimiento del capital humano y educación de los clientes. También busca mejorar la productividad de la industria de la construcción y transformarla en un referente internacional. El programa Construye 2025 propone alcanzar los desafíos pendientes en Chile: crecer al ritmo de los últimos 30 años (4,1\% per cápita) para alcanzar a España en 2025 y a EE. UU. en 2035 y ampliar las oportunidades para reducir las enormes desigualdades actuales. Para cumplir estos objetivos, resulta adecuada la adaptación de los modelos que ya están en funcionamiento, como el del caso español. A modo de ejemplo de todo el trabajo que queda por hacer en Chile, se debe señalar que hoy en día solo hay siete sitios para disposición final de residuos de construcción en toda la Región Metropolitana y aproximadamente cinco más en todo Chile (BRITO, 2018).

\section{Modelos de cuantificación de RCD}

Varios modelos se han establecido para determinar la cantidad de residuos del proyecto, entre ellos, el SuperDrecksKëscht fir Betriber (OEKOSERVICE..., 2002), que propone la cuantificación de RCD en tipos y volúmenes producidos. Por su parte, la Universidad Técnica Nacional de Atenas (NTUA) desarrolló un modelo matemático indicativo para estimar la cantidad de residuos generados (KOURMPANIS et al., 2008). SMARTWaste TM es otro método de cuantificación aplicado en el Reino Unido; se basa en datos obtenidos en experiencias previas y calcula los volúmenes en 13 categorías (como cerámica, hormigón, palets de madera, etc). Otros modelos de cuantificación, desarrollo matemático y software se pueden encontrar en Cheng y Ma (2013) y Wu et al. (2014).

En el trabajo de Wu et al. (2014) se repasan 57 propuestas internacionales para clasificar y cuantificar los RCD, estructurándolas en visitas a obra, cálculo de generación media, análisis de flujo de materiales, sistemas por variables y sistemas acumulativos.

\section{Visitas a obra}

En las visitas a obras se realizan encuestas reales y se emplean métodos directos e indirectos. En el primer caso es necesario pesar los residuos o medir su volumen.

\section{Cálculo de generación media}

Con este método se definen producciones en $\mathrm{kg} / \mathrm{m}^{2}$ construido o $\mathrm{m}^{3} / \mathrm{m}^{2}$ construido. Estos pueden ser per cápita, por costo del proyecto o por área construida. Como presentado en los trabajos de McBean y Fortin (1993), la debilidad de esta estimación es que la producción fluctúa con la economía y no con la población, que es prácticamente constante a lo largo de los años. Lo mismo ocurre si sólo se toma en consideración el valor de los proyectos (YOST; HALSTEAD, 1996) o el área construida, casos en 
los que se recurre a simplificaciones en las que tan solo se mide un aspecto del proyecto (VILLORIASÁEZ; PORRAS-AMORES; DEL RÍO MERINO, 2015).

\section{Análisis de flujo de materiales}

En este caso, los estudios se basan en análisis de flujo de materiales de construcción a nivel de país (COCHRAN; TOWNSEND, 2010), conforme la siguiente Ecuación 1:

$\mathrm{CW}=\mathrm{M} \times \mathrm{Wc}$

Donde:

$\mathrm{Cw}=$ cantidad de residuos producidos en la construcción en todo el país;

$\mathrm{M}=$ cantidad de material comprado en todo el país; y

Wc = porción media que termina convirtiéndose en residuo en todo el país.

Siguiendo el principio de balance de masa, Li et al. (2016) calcularon que la suma en peso de los materiales de construcción más su embalaje equivalen a los elementos de construcción objetivo y sus residuos de construcción, lo cual facilita su cuantificación.

\section{Sistemas por variables}

La generación de residuos en las obras depende de un gran número de variables, tales como indicadores económicos, superficie construida, condiciones del lugar de trabajo, etc. Por su parte, Wimalasena, Ruwanpura y Hettiaratchi (2010) proponen las siguientes: actividad específica, mano de obra y equipos, materiales y su almacenamiento, ubicación de la obra, clima y, por último, políticas empresariales. También en la línea de cálculo basado en variables están los trabajos de Aguirre et al. (2005) y Mokhtar et al. (2011), que identifican las siguientes variables principales para controlar la cantidad de residuos esperable: los métodos de construcción, el tamaño del proyecto, el tipo de construcción, la forma de almacenamiento de materiales, el error humano y los problemas técnicos.

\section{Sistemas acumulativos}

Los sistemas acumulativos son los sistemas de cálculo de residuos más mencionados en la literatura (COELHO; BRITO, 2011; LLATAS, 2011; SOLÍS-GUZMAN et al., 2009; MERCADER-MOYANO; RAMIREZ-DEARELLANO, 2013). En ellos es necesario aplicar un método de clasificación sistemática de los trabajos de construcción que son la base del cálculo y estimaciones para cada material de construcción. Estos sistemas ofrecen una metodología efectiva para determinar los residuos y definir estrategias específicas para cada naturaleza; se combinan con programas de ordenador o tablas de cálculo.

\section{Metodología propuesta}

La propuesta metodológica se basa en la última categoría expuesta en la sección anterior, correspondiente a los sistemas acumulativos. SolísGuzmán et al. (2009) han desarrollado un modelo de cuantificación para estimar el tipo y la cantidad de residuos generados en edificios nuevos, demolición y renovaciones. El código de clasificación utilizado es el mismo que usan los ingenieros de edificación españoles para obtener la lista de cantidades, es por esto que el modelo es fácil de entender e implementar por parte de los técnicos (MARRERO; RAMIREZ-DE-ARELLANO, 2010). En 2013, se implementaron estos sistemas en la Mancomunidad de los Alcores, Sevilla (PÉREZCARMONA; MARRERO; SOLÍS-GUZMÁN, 2013), y, más adelante, en la Mancomunidad de Écija, también en Sevilla. El modelo se adaptó con éxito a la obra civil, más específicamente en la construcción de carreteras (SOLÍS-GUZMÁN; MARRERO; GUISADO GARCIA, 2014).

Para poder adaptar el modelo español a Chile es necesario realizar una buena cuantificación de los RCD esperados, cuestión imprescindible en la que se centró la presente propuesta, y una clasificación y definición de estrategias para aplicar el principio de jerarquía: prevención, reutilización, reciclado y eliminación controlada. Para que ocurra un cambio en la gestión, ésta debe reflejarse en el control de los costos, con lo cual los presupuestos de los proyectos son la vía principal para introducir estrategias de reducción de impactos económicos y ambientales.

Todo lo anterior se recoge en el Real Decreto (RD), 105/2008 (ESPAÑA, 2008), por medio del documento denominado Estudio de Gestión de Residuos (EGR), que debe contener una estimación de la cantidad de residuos previstos, planes de acción para la prevención, medidas de separación, planos de las instalaciones, pliego de prescripciones técnicas particulares del proyecto, valoración del costo previsto para la gestión de los RCD, un inventario de residuos peligrosos a generar $\mathrm{y}$, por último, aseguramiento del envío de todos los residuos a gestores autorizados. A continuación, se describe cómo se abordan la cuantificación y el presupuesto para, finalmente, ver la aplicabilidad del EGR en Chile a partir de un caso de estudio. 


\section{Clasificación de los RCD: lista europea de residuos}

Para realizar la clasificación el RD exige la caracterización y codificación de los RCD de acuerdo con la lista europea de residuos (LER) publicada por Orden MAM/304/2002 (ESPAÑA, 2002), de 8 de febrero, por la que se publican las operaciones de valorización y eliminación de residuos y la LER o sus modificaciones posteriores (ESPAÑA, 2002).

\section{Modelo de estimación de la cantidad de residuos}

Para poder determinar la cantidad de RCD esperados en la obra, se deben identificar los elementos generadores de residuos. En su mayoría serán materiales de construcción, aunque también generan residuos los trabajos previos de limpieza, demolición y movimiento de tierra.

Para identificar los elementos generadores de residuos, el modelo se basa en los datos recogidos en el presupuesto del proyecto, en el que, una vez definidas todas sus unidades de obra, se le asigna una cantidad y tipo de residuo a cada elemento generado. El modelo propuesto utiliza la base de costes de la construcción de Andalucía (BCCA) para su clasificación (COMUNIDAD..., 2017). Su uso más extendido es para estimar el costo de la construcción de viviendas y es obligatorio su empleo en desarrollos públicos en Andalucía, España.

La BCCA divide las unidades de trabajo en una organización jerárquica. El nivel más alto es el proyecto terminado y las siguientes divisiones se denominan capítulos y coinciden con un proceso de construcción: demolición, movimiento de tierras, cimientos, disposición de agua, estructura, partición, techo, instalación, aislamiento, acabado, carpintería, vidrio y poliéster, revestimiento, decoración, urbanización, seguridad y gestión de residuos.

\section{Cuantificación de residuos}

Una vez que todos los elementos que generan desechos están identificados y clasificados, los desechos se pueden calcular en función del elemento original con la siguiente ecuación (Ec. 2), utilizando tres coeficientes de transformación (SOLÍS-GUZMÁN et al., 2009).
$\mathrm{QRi}=\mathrm{Qi} \times \mathrm{CRi} \times \mathrm{CCi} \times \mathrm{CTi}$

Ec. 2

Donde:

$\mathrm{QRi}=$ cantidad de residuos que genera el elemento constructivo o material "i" (EC/Mi);

$\mathrm{Qi}=$ cantidad de EC/Mi original;

$\mathrm{CRi}=$ porcentaje del EC/Mi original que se convierte en residuo;

$\mathrm{CCi}$ = coeficiente para cambiar las unidades del EC/Mi a unidades de elementos de desecho; y

$\mathrm{CTi}$ = coeficiente que toma en cuenta el cambio de volumen del material.

A continuación, se detalla, a modo de ejemplo, el cálculo de los coeficientes CC, CR y CT de un muro de ladrillo, considerando que (Figura 4):

(a) las dimensiones del ladrillo son 24,00 × 11,50 $\times 7,00 \mathrm{~cm}$;

(b) durante la demolición, el ladrillo se esponja un 30\%; $\mathrm{y}$

(c) la superficie ejecutada es de $630 \mathrm{~m}^{2}$.

En la Figura 5 se detalla el cálculo del volumen de RCD. Se identifican los $\mathrm{m}^{2}$ de superficie ejecutada (medición de origen) y los $\mathrm{m}^{3}$ de residuos de ladrillos y mortero de pega (volumen esponjado) que se retiran (medición de destino).

\section{Determinación de coeficientes}

A modo de ejemplo, la etapa de trabajo preliminar está compuesta por movimiento de tierras, limpieza y tala de árboles. La aplicación de la metodología actual al trabajo de movimiento de tierras, excavación de terrenos, desmonte, etc. se convierte en un residuo y, por lo tanto, el coeficiente CR es igual a 1,00 cuando el suelo no se usa como relleno. El coeficiente CC también es igual a 1,00 porque la unidad de medida del elemento $\left(\mathrm{m}^{3}\right.$ de suelo) es la misma en el proyecto de construcción que en el plan de gestión de residuos. Finalmente, CT toma en cuenta el cambio del volumen del suelo después de la excavación, que tiene lugar durante el desmonte y la apertura de cajas, fosas, zanjas, etc. El coeficiente es 1,25 , un valor de expansión promedio para diferentes tipos de suelo. En el trabajo requerido para rellenos sanitarios, rellenos, etc., la hipótesis es que se desperdicia el $1 \%$ del suelo durante la manipulación, y CR es, por lo tanto, de 0,01. Más ejemplos son recogidos en el Cuadro 1 . 
Figura 4 - Ejemplo RCD ladrillo

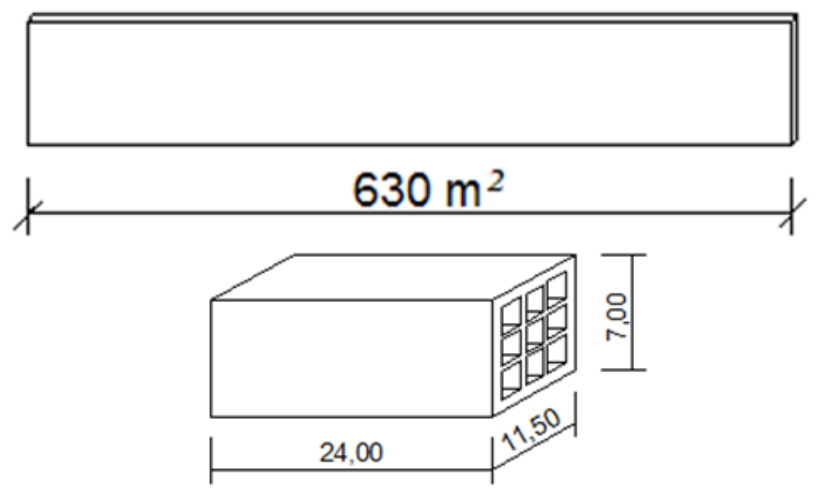

Figura 5 - Ejemplo RCD ladrillo y cálculo de coeficientes
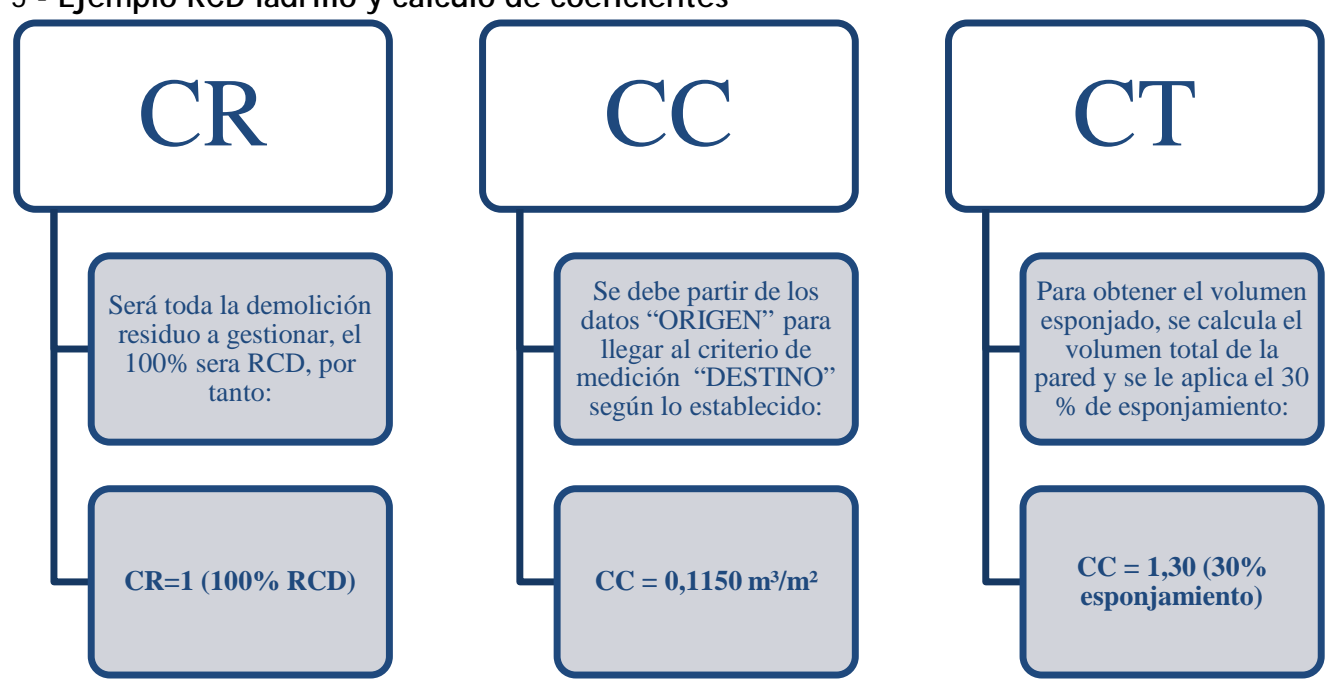

Cuadro 1 - Coeficientes CR, CC y CT para obras de urbanización ejemplos caso estudio

\begin{tabular}{|c|c|c|c|c|c|}
\hline Elemento constructivo & Origen residuo & $\begin{array}{l}\text { Residuo } \\
\text { generado }\end{array}$ & $C R$ & $C C$ & $C T$ \\
\hline \multirow{6}{*}{$\begin{array}{l}\text { Pozo registro } D=110 \mathrm{~mm} \mathrm{~h}=2,5 \mathrm{~m} \\
\text { (ud) }\end{array}$} & Hormigón & Pérdidas & 0,05 & 0,45 & 1,10 \\
\hline & Tierras & Escavación & 1,00 & 2,50 & 1,25 \\
\hline & Ladrillo & Pérdidas & 0,06 & 1,77 & 1,30 \\
\hline & Mortero & Pérdidas & 0,05 & 0,68 & 1,10 \\
\hline & Palet de madera & Envases & 0,05 & 1,56 & 1,00 \\
\hline & Plástico & Envases & 1,00 & 0,537 & 1,00 \\
\hline Pintura reflexiva marcas viales $(\mathrm{m})$ & Envase contenedor & Envases & 1,00 & 0,008 & 1,00 \\
\hline Pintura reflexiva marcas viales $(\mathrm{m})$ & Palet de madera & Envases & 0,05 & 0,00025 & 1,00 \\
\hline Pintura reflexiva marcas viales $(\mathrm{m})$ & Plástico & Envases & 1,00 & $1,28 \times 10^{-5}$ & 1,00 \\
\hline Circuito alumbrado público $(\mathrm{m})$ & Cobre & Pérdidas & 0,05 & 0,829 & 1,00 \\
\hline Circuito alumbrado público $(\mathrm{m})$ & Bobina de madera & Envases & 0,05 & 0,001 & 1,00 \\
\hline $\begin{array}{l}\text { Excavación de tierra en formación } \\
\text { de cajas }\left(\mathrm{m}^{3}\right)\end{array}$ & Tierras & Excavación & 1,00 & 1,00 & 1,25 \\
\hline Calzada asfáltica $(t)$ & Asfalto & Pérdidas & 0,05 & 1,00 & 1,00 \\
\hline $\begin{array}{l}\text { Tubería para agua de } P E H D \\
D=90 \mathrm{~mm}(\mathrm{~m})\end{array}$ & PEHD & Pérdidas & 0,05 & 0,00213 & 1,00 \\
\hline $\begin{array}{l}\text { Tubería para agua de } P E H D \\
D=90 \mathrm{~mm}(\mathrm{~m})\end{array}$ & Arena & Pérdidas & 0,01 & 0,065 & 1,00 \\
\hline
\end{tabular}

Fuente: Puerto (2011). 


\section{Valoración del costo previsto}

Una vez definida de forma sistemática la cuantificación de residuos, el siguiente paso es valorar el costo previsto o su presupuesto, y para ello se emplea la misma clasificación sistemática. La estructura de costos de la BCCA es arborescente y jerarquizada (Figura 6). La base de la pirámide está formada por los precios de suministro (PSU), que conectan directamente el sistema con los mercados: mano de obra, materiales, maquinaria, subcontratos, etc. En el vértice de la estructura, se sitúan los importes de contrato (IC) y costos exógenos (CE), completándose con niveles intermedios: precios básicos (PB), precios unitarios simples (PUS) y, por último, el importe de ejecución material (IEM) más los costos indirectos (CI).

En el Cuadro 2 se muestra un ejemplo de precios básicos que componen el precio unitario
17MMM00120 (t) Retirada de residuos de madera, papel y cartón a planta de valorización a 15 km de distancia.

El capítulo de la BCCA titulado "Gestión de Residuos” está formado por los siguientes subcapítulos: metales, asfalto, hormigón, material de aislamiento, madera, papel, suelos, entre otros (Cuadro 3), y cuenta con más de 70 precios unitarios de gestión de residuos.

Para obtener un presupuesto adecuado es necesario definir características adicionales. Las unidades de obra dentro de los sub-capítulos se pueden asignar a las siguientes condiciones: residuos depositados en los contenedores en obra, residuos cargados en camiones en obra, residuos enviados al centro de reciclaje sin descarga y residuos enviados al centro de reciclaje, incluyendo la descarga (MARRERO; RAMIREZ-DE-ARELLANO, 2010).

\section{Figura 6 - Pirámide de precios}

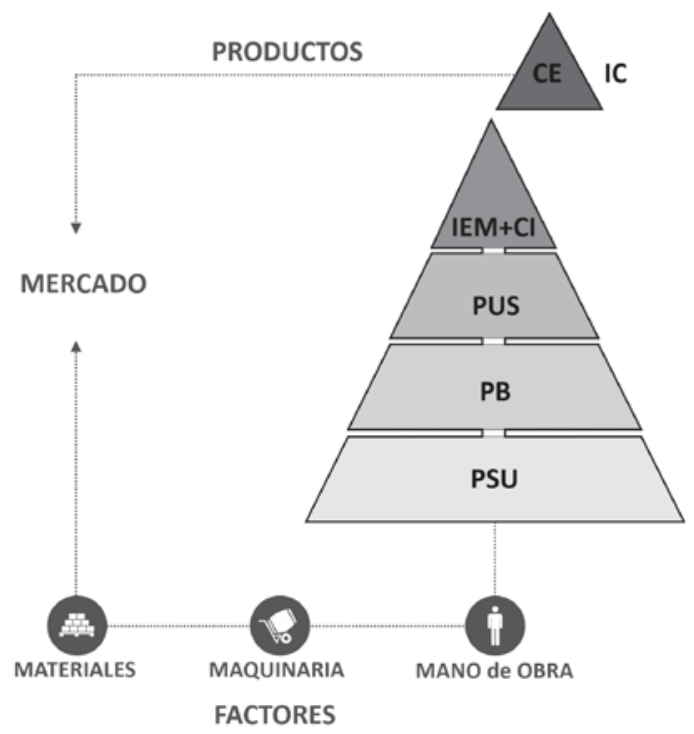

Fuente: Solís-Guzman, Marrero y Guisado Garcia (2014).

\section{Cuadro 2 - Ejemplo de precio unitario de RCD}

\begin{tabular}{|c|c|c|c|c|c|c|}
\hline \multicolumn{2}{|c|}{ 17MМM00120 } & $\mathbf{T}$ & \multicolumn{4}{|c|}{$\begin{array}{c}\text { Retirada de residuos de madera, papel y cartón a planta de } \\
\text { valorización } 15 \mathrm{~km} \text {. }\end{array}$} \\
\hline \multicolumn{7}{|c|}{$\begin{array}{l}\text { Retirada de residuos de madera en obra a planta de valorización situada a una distancia máxima de } 15 \\
\text { km. Consiste en: transporte interior, carga, transporte a planta, descarga y canon de gestión. Medido } \\
\text { el peso en báscula puesto en planta. }\end{array}$} \\
\hline \multicolumn{7}{|c|}{ Medido el peso en báscula descargado en planta de tratamiento } \\
\hline Código & & & epto & Cantidad & Precio unitario (€) & Importe (€) \\
\hline EM00100 & t Car & & $\begin{array}{l}\text { de residuos de } \\
\text { ra }\end{array}$ & 1,00 & 6,00 & 6,00 \\
\hline ME00300 & & & gadora & 0,020 & 23,87 & 0,48 \\
\hline MK00100 & & $\overline{\mathrm{Ca}}$ & basculante & 0,300 & 25,60 & 7,68 \\
\hline
\end{tabular}

Fuente: Comunidad Autónoma del BCCA (2017). 
Cuadro 3 - Clasificación de precios básicos de acuerdo con la BCCA

\begin{tabular}{|l|l|}
\hline \multicolumn{2}{|c|}{ E. RESIDUOS (restos, pérdidas, escombros, etc.) } \\
\hline EA. & Metales y aleaciones \\
\hline EF. & Asfalto, alquitrán y otros productos alquitranados \\
\hline EH. & Hormigones, ladrillos, tejas, materiales cerámicos y materiales derivados del yeso \\
\hline EI. & Materiales de aislamiento \\
\hline EM. & Maderas, papeles, cartones, plásticos, sintéticos y vidrios \\
\hline ER. & Residuos metálicos \\
\hline ET. & Terrenos \\
\hline EW. & Varios \\
\hline
\end{tabular}

Fuente: Solís-Guzman, Marrero y Guisado (2014).

Los costos negativos son permitidos en la estructura de costos de la BCCA, con el fin de representar la reutilización y el reciclado, en el caso particular de que los residuos sean reutilizados en la propia obra o vendidos a terceros para su reciclaje o reutilización (MARRERO et al., 2011).

\section{Caso de estudio}

El caso de estudio es una adaptación del modelo metodológico español y consiste en la construcción de 17 viviendas unifamiliares durante la etapa de urbanización del terreno ubicado en el condominio Nuevo Mundo, Temuco, Región de la Araucanía, que tiene una superficie de 2.471,25 $\mathrm{m}^{2}$ para urbanizar (Figura 7).

El proyecto de urbanización incluye las siguientes partidas:

(a) obras de viarios en las que se incluyen los movimientos de tierra de explanación, contención y pavimentación de calzadas, construcción y encintado de soleras y canalizaciones en el subsuelo de las vías o de soleras;

(b) obras de saneamiento, que incluyen colectores generales y parciales, acometidas y sumideros; $y$

(c) obras para la instalación y el funcionamiento de los servicios públicos de suministro de agua, energía eléctrica, telefonía y telecomunicaciones, así como el alumbrado público.

\section{Estimación de las cantidades de RCD que generará el proyecto}

Tras aplicar la metodología planteada, se obtuvo la previsión de los residuos detallados en el Cuadro 4, los cuales han sido clasificados según la LER, cuya cuantificación consta en volumen $\left(\mathrm{m}^{3}\right)$ y en peso $(\mathrm{t})$. Nota: el asterisco en el código LER indica que el residuo es peligroso o potencialmente peligroso.

\section{Medidas para prevenir, reutilizar y reciclar}

Con la previsión de cantidades de RCD que se generarán, se definen las medidas para su correcta gestión en obra. Lo principal es tener previsto un lugar de acopio de los materiales y de los RCD que se irán generando, quedando registrados en un plano que forma parte del resto de la documentación del proyecto (Figura 8).

El plano del lugar de acopio de material también es requisito en la normativa española para todo proyecto que sea visado. De ese modo, se consigue que las tareas se realicen de forma ordenada, controlando en todo momento la disponibilidad de los distintos materiales de construcción, evitando posibles desperfectos por golpes, derribos o contaminación y reduciendo los excesos de residuos. En esta línea están disponibles los trabajos de mejoras en la gestión en obra de Souza et al. (2004) y de gestión de los trabajos en obra a medio terminar de Formoso et al. (2017).

\section{Valorización de los costos de gestión de RCD}

Una vez obtenidas las cantidades a gestionar, se le aplican los costos de gestión según la naturaleza de los residuos que se han previsto. Para ello, se han usado los precios desarrollados en la BCCA para el capítulo de gestión de RCD. Se puede analizar el presupuesto en el Cuadro 5, elaborado en euros, que muestra que la partida de gestión de metales presenta un costo negativo. Esto se traduce en un ingreso de capital al presupuesto porque la partida de metales y aleaciones está planificada en el estudio de gestión como una partida valorizable, dado que dichos materiales serán vendidos para su reciclaje por una empresa externa. Cada una de las unidades de obra en el capítulo del presupuesto “Gestión de RCD” define explícitamente el destino final, ya sea reciclaje, reutilización o eliminación en vertedero controlado. 
Figura 7 - Urbanización del caso de estudio

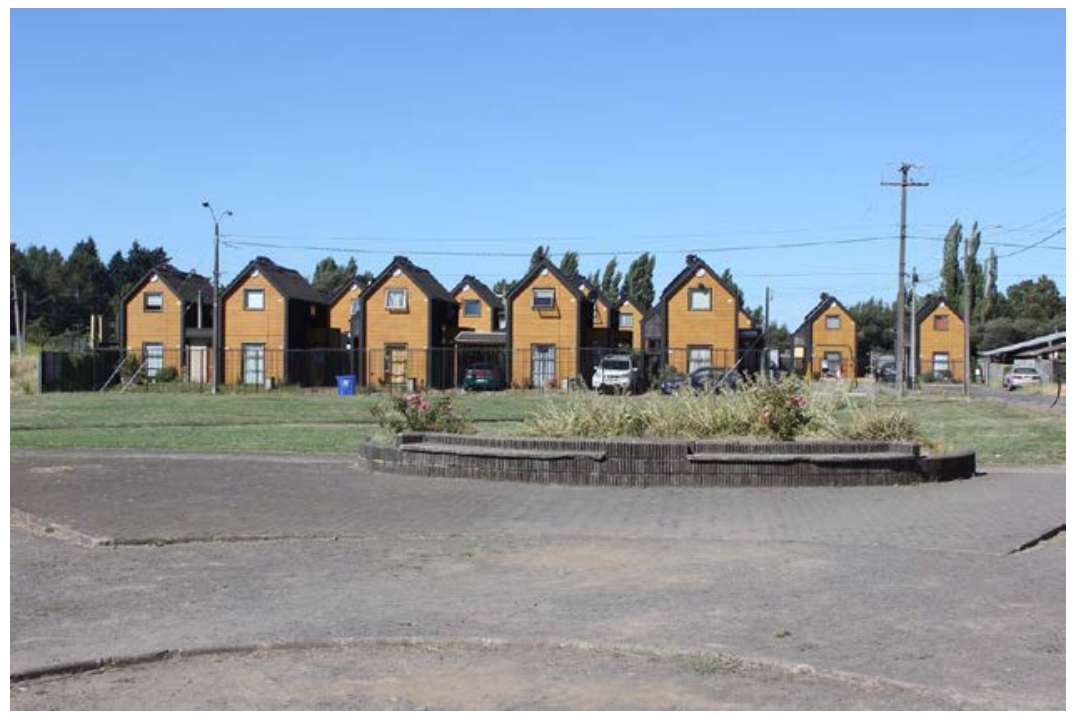

Fuente: Universidad del Bio-Bio (2018).

Cuadro 4 - Clasificación y cuantificación de los RCD generados

\begin{tabular}{|c|c|c|c|}
\hline $\begin{array}{l}\text { Código } \\
\text { LER }\end{array}$ & Concepto & Volumen $\left(\mathrm{m}^{3}\right)$ & Peso (t) \\
\hline 17 & \multicolumn{3}{|c|}{$\begin{array}{l}\text { RESIDUOS DE LA CONSTRUCCIÓN Y DEMOLICIÓN (INCLUIDA LA } \\
\text { TIERRA EXCAVADA DE ZONAS CONTAMINADAS) }\end{array}$} \\
\hline 1701 & \multicolumn{3}{|c|}{ Hormigón, ladrillos, tejas y materiales cerámicos } \\
\hline 170101 & Hormigón & 26,726 & 64,142 \\
\hline 170102 & Ladrillos & 4,221 & 5,698 \\
\hline 1702 & \multicolumn{3}{|c|}{ Madera, vidrio y plástico } \\
\hline 170203 & Plástico & 0,098 & 0,138 \\
\hline 1703 & \multicolumn{3}{|c|}{ Mezclas bituminosas, alquitrán de hulla y otros productos alquitranados } \\
\hline 170302 & $\begin{array}{l}\text { Mezclas bituminosas distintas de las especificadas } \\
\text { en el codigo } 170301 \text { (sin alquitrán de hulla) }\end{array}$ & 10,155 & 11,170 \\
\hline 1704 & \multicolumn{3}{|c|}{ Metales (incluidas sus aleaciones) } \\
\hline 170401 & Cobre & 0,001 & 0,009 \\
\hline 170402 & Aluminio & 0,009 & 0,023 \\
\hline 170405 & Acero & 0,0001 & 0,0005 \\
\hline 1705 & \multicolumn{3}{|c|}{ Tierra (incluida la excavada de zonas contaminadas), piedras y lodos de drenaje } \\
\hline 170504 & $\begin{array}{l}\text { Tierras y piedras que no contienen sustancias } \\
\text { peligrosas }\end{array}$ & $1.442,114$ & $2.451,593$ \\
\hline 15 & \multicolumn{3}{|c|}{$\begin{array}{l}\text { RESIDUOS DE ENVASES; ABSORBENTES, TRAPOS DE LIMPIEZA; } \\
\text { MATERIALES DE FILTRACIÓN Y ROPAS DE PROTECCIÓN NO } \\
\text { ESPECIFICADOS EN OTRA CATEGORÍA }\end{array}$} \\
\hline 1501 & \multicolumn{3}{|c|}{ Maderas, papeles, cartones, plásticos, sintéticos y vidrios } \\
\hline 150101 & Envases de papel y cartón & 0,076 & 0,083 \\
\hline 150102 & Envases de plástico & 0,033 & 0,046 \\
\hline 150103 & Envases de madera & 0,144 & 0,576 \\
\hline $150110^{*}$ & $\begin{array}{l}\text { Envases que contienen restos de sustancias } \\
\text { peligrosas. }\end{array}$ & 0,009 & 0,009 \\
\hline TOTAL & & $1.483,576$ & $2.533,480$ \\
\hline
\end{tabular}


Figura 8 - Plano de ubicación de acopios y contenedores para la separación

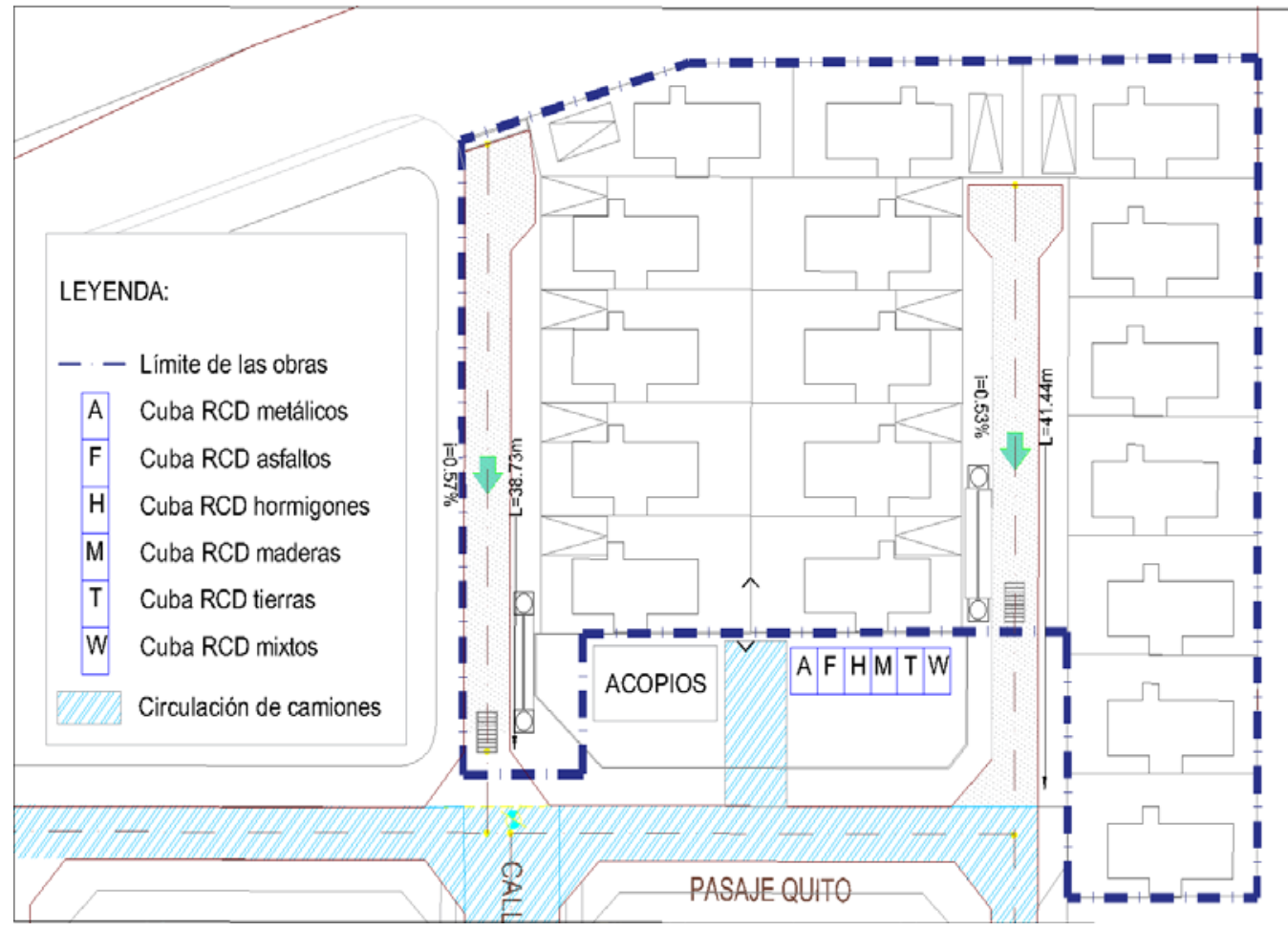

Cuadro 5 - Presupuesto de los RCD generados (parte 1 de 2) (Continúa...)

\begin{tabular}{|c|c|c|c|c|c|}
\hline \multicolumn{2}{|c|}{ Identificación } & \multicolumn{2}{|c|}{ Dimensiones } & \multicolumn{2}{|c|}{ Importe } \\
\hline Código & Concepto & Medición & Precio & Parcial & Total \\
\hline 17A. & \multicolumn{5}{|c|}{ METALES Y ALEACIONES } \\
\hline \multirow{3}{*}{ 17AAA00140 } & \multirow{2}{*}{\multicolumn{5}{|c|}{$\begin{array}{l}\text { t RETIRADA RESIDUOS ALUMINIO N.P. DIST. MÁX. } 15 \mathrm{~km} \\
\text { Retirada de residuos de aluminio en obra de nueva planta situada a una distancia } \\
\text { máxima de } 15 \mathrm{~km} \text {. Consiste en: transporte interior, carga, transporte y descarga } \\
\text { en almacén. Medido el peso en báscula puesto en almacén. }\end{array}$}} \\
\hline & & & & & \\
\hline & & 0,023 & $-72,27$ & $-1,68$ & \\
\hline \multirow{3}{*}{ 17АВC00140 } & \multicolumn{5}{|c|}{ t RETIRADA RESIDUOS COBRE N.P. DIST. MÁX. 15 km } \\
\hline & \multicolumn{5}{|c|}{$\begin{array}{l}\text { Retirada de residuos de cobre en obra de nueva planta situada a una distancia } \\
\text { máxima de } 15 \mathrm{~km} \text {. Consiste en: transporte interior, carga, transporte y descarga } \\
\text { en almacén. Medido el peso en báscula puesto en almacén. }\end{array}$} \\
\hline & & 0,01 & $-72,27$ & $-0,64$ & \\
\hline \multirow{3}{*}{ 17АНА00140 } & \multicolumn{5}{|c|}{ t RETIRADA RESIDUOS ACERO N.P. DIST. MÁX. 15 km } \\
\hline & \multicolumn{5}{|c|}{$\begin{array}{l}\text { Retirada de residuos de acero en obra de nueva planta situada a una distancia } \\
\text { máxima de } 15 \mathrm{~km} \text {. Consiste en: transporte interior, carga, transporte y descarga } \\
\text { en almacén. Medido el peso en báscula puesto en almacén. }\end{array}$} \\
\hline & & 0,000 & $-67,69$ & $-0,03$ & \\
\hline \multirow{2}{*}{\multicolumn{6}{|c|}{\begin{tabular}{l|l}
\multicolumn{2}{|c}{ TOTAL SUBCAPÍTULO 17A: METALES Y ALEACIONES } \\
& ASFALTOS
\end{tabular}}} \\
\hline & & & & & \\
\hline \multirow{3}{*}{ 17FSS00300 } & \multicolumn{5}{|c|}{ t RETIRADA EN CONTENEDOR DE RESIDUOS ASFÁLTICOS } \\
\hline & \multicolumn{5}{|c|}{$\begin{array}{l}\text { Retirada de residuos de asfálticos en obra a planta de valorización situada a una } \\
\text { distancia máxima de } 15 \mathrm{~km} \text {. Consiste en: transporte interior, carga, transporte a } \\
\text { planta, descarga y canon de gestión. Medido el volúmen esponjado. }\end{array}$} \\
\hline & & 11,17 & 15,21 & 169,90 & \\
\hline
\end{tabular}


Cuadro 5 - Presupuesto de los RCD generados (parte 2 de 2) (continuación)

\begin{tabular}{|c|c|c|c|c|c|}
\hline \multicolumn{5}{|c|}{ TOTAL SUBCAPÍTULO 17F: ASFALTOS } & $€ 169,90$ \\
\hline 17H. & \multicolumn{5}{|c|}{ HORMIGONES, LADRILLOS, TEJAS, ETC. } \\
\hline \multirow{3}{*}{ 17HCL00300 } & \multicolumn{5}{|c|}{$\mathrm{m}^{3}$ RETIRADA EN CONTENEDOR DE RESIDUOS DE LADRILLOS } \\
\hline & \multicolumn{5}{|c|}{$\begin{array}{l}\text { Retirada de residuos de materiales de ladrillo mezclados en obra a planta de } \\
\text { valorización situada a una distancia máxima de } 15 \mathrm{~km} \text {. Consiste en: transporte } \\
\text { interior, carga, transporte a planta, descarga y canon de gestión. Medido el } \\
\text { volúmen esponjado }\end{array}$} \\
\hline & & 4,22 & 13,49 & 56,94 & \\
\hline \multirow{3}{*}{ 17НHН00300 } & \multicolumn{5}{|c|}{$\begin{array}{lccccccc}\text { m }^{3} & \text { RETIRADA } & \text { RESIDUOS } & \text { DE } & \text { HORMIGÓN } & \text { A } & \text { PLANTA } & \text { DE } \\
\text { VALORIZACIÓN. } 15 \mathrm{~km} & & & & & \end{array}$} \\
\hline & \multicolumn{5}{|c|}{$\begin{array}{l}\text { Retirada de residuos de hormigón en obra a planta de valorización situada a una } \\
\text { distancia máxima de } 15 \mathrm{~km} \text {. Consiste en: transporte interior, carga, transporte a } \\
\text { planta, descarga y canon de gestión. Medido el volúmen esponjado }\end{array}$} \\
\hline & & 26,73 & 21,67 & 579,15 & \\
\hline \multicolumn{6}{|c|}{\begin{tabular}{|l|l|c|}
\multicolumn{2}{|c|}{ TOTAL SUBCAPÍTULO 17H: HORMIGONES, LADRILLOS, TEJAS, ETC. } & $€$ 636,09 \\
\end{tabular}} \\
\hline 17M. & \multicolumn{5}{|c|}{$\begin{array}{l}\text { MADERAS, PAPELES, CARTONES, PLÁSTICOS, SINTÉTICOS Y } \\
\text { VIDRIOS }\end{array}$} \\
\hline \multirow{5}{*}{ 17MMM00120 } & \multirow{2}{*}{\multicolumn{5}{|c|}{$\begin{array}{l}\text { t RETIRADA RESIDUOS DE MADERA, PAPEL Y CARTÓN A PLANTA DE } \\
\text { VALORIZACIÓN. } 15 \mathrm{~km} \\
\text { Retirada de residuos de madera en obra a planta de valorización situada a una } \\
\text { distancia máxima de } 15 \mathrm{~km} \text {. Consiste en: transporte interior, carga, transporte a } \\
\text { planta, descarga y canon de gestión. Medido el peso en báscula puesto en planta. }\end{array}$}} \\
\hline & & & & & \\
\hline & $\begin{array}{l}\text { ENVASES PAPEL Y } \\
\text { CARTÓN }\end{array}$ & 0,083 & & & \\
\hline & \multirow[t]{2}{*}{ ENVASES MADERA } & 0,58 & & & \\
\hline & & 0,66 & 15,66 & 10,32 & \\
\hline \multirow{5}{*}{ 17MМР00140 } & \multirow{2}{*}{\multicolumn{5}{|c|}{$\begin{array}{l}\text { t RETIRADA RESIDUOS PLÁSTICOS Y SINTÉTICOS, DIST. MÁX. } 15 \text { km } \\
\text { MEC } \\
\text { Retirada de residuos plásticos y sintéticos realizada en camión basculante a una } \\
\text { distancia máxima de } 15 \mathrm{~km} \text {, incluso carga con medios mecánicos. Medido el peso } \\
\text { en báscula. }\end{array}$}} \\
\hline & & & & & \\
\hline & PLÁSTICOS & 0,14 & & & \\
\hline & \multirow[t]{2}{*}{$\begin{array}{ll}\text { ENVASES } & \text { DE } \\
\text { PLÁSTICO } & \\
\end{array}$} & 0,05 & & & \\
\hline & & 0,18 & 69,18 & 12,74 & \\
\hline \multicolumn{5}{|c|}{$\begin{array}{l}\text { TOTAL SUBCAPÍTULO 17H:MADERAS, PAPELES, CARTONES, } \\
\text { PLÁSTICOS, SINTÉTICOS Y VÍDRIOS }\end{array}$} & $€ 23,06$ \\
\hline 17T. & \multicolumn{5}{|l|}{ TERRENOS } \\
\hline \multirow{3}{*}{ 17TTT00120 } & \multicolumn{5}{|c|}{$\begin{array}{l}\mathrm{m}^{3} \text { RETIRADA DE TIERRAS INERTES N.P. A VERTEDERO AUTORIZADO } \\
15 \mathrm{~km}\end{array}$} \\
\hline & \multicolumn{5}{|c|}{$\begin{array}{l}\text { Retirada de tierras inertes en obra de nueva planta a vertedero autorizado situado } \\
\text { a una distancia máxima de } 15 \mathrm{~km} \text {. Consiste en: selección, carga, transporte, } \\
\text { descarga y canon de vertido. Medido el volumen esponjado. }\end{array}$} \\
\hline & & 1442,11 & 10,13 & 14608,61 & \\
\hline \multicolumn{5}{|c|}{ TOTAL SUBCAPÍTULO 17H: TERRENOS } & $€ 14.608,61$ \\
\hline 17W. & \multicolumn{5}{|l|}{ VARIOS } \\
\hline \multirow{3}{*}{ 17WWW00100 } & \multicolumn{5}{|c|}{ t RETIRADA EN CONTENEDOR DE $3 \mathrm{~m}^{3}$ DE RESIDUOS PELIGROSOS } \\
\hline & \multicolumn{5}{|c|}{$\begin{array}{l}\text { Retirada en contenedor de } 3 \mathrm{~m}^{3} \text { de envases contaminados con sustancias } \\
\text { peligrosas en obra a planta de valorización situada a una distancia máxima de } 15 \\
\text { km. Consiste en: transporte interior, carga, transporte a planta, descarga y canon } \\
\text { de gestión. Medido el peso en báscula puesto en planta. }\end{array}$} \\
\hline & & 0,009 & 41,54 & 0,37 & \\
\hline \multicolumn{5}{|c|}{ TOTAL SUBCAPÍTULO 17W: VARIOS } & $€ 0,37$ \\
\hline \multicolumn{5}{|c|}{ TOTAL DEL PRESUPUESTO DE GESTIÓN DE RCD } & $€ 15.435,68$ \\
\hline
\end{tabular}


Es preciso enfatizar que los costos aplicados se rigen por los procesos de gestión que se aplican en España, concretamente en Andalucía, Comunidad Autónoma de la Base de Costes de la Construcción de Andalucía (BCCA). Los resultados aquí obtenidos son de carácter meramente orientativo, siendo necesario un estudio pormenorizado de las posibilidades de gestión de residuos existentes en Chile con sus correspondientes costos.

La finalidad de este apartado es mostrar de qué forma una correcta separación en origen y gestión de los RCD generados (con la prevención, reutilización y reciclaje planteados en el estudio) contribuye a cuidar el medioambiente, genera importantes ahorros en la gestión de RCD e incluso puede llegar a generar ingresos en el proyecto de ejecución.

\section{Resultados y discusión}

\section{Análisis}

Tras la aplicación del modelo Alcores al caso de estudio, se procederá a analizar las proporciones de RCD. Para una mejor percepción, en la Figura 9 se han representado los porcentajes de cada tipo de residuo, a excepción de las tierras, actividad prioritaria en los proyectos de urbanización, dado que representan el $97 \%$ del total de los residuos generados, principalmente durante el proceso de excavación. Esto hace que sea indispensable realizar, desde la fase de diseño del proyecto, un estudio para la reutilización de las tierras, lo que producirá un gran ahorro en los costos de gestión de RCD y el consumo de recursos naturales, disminuyendo el impacto ambiental que ocasionan los proyectos de urbanización.
Por lo tanto, es posible afirmar que, por medio de la reutilización de tierras, se puede generar un doble ahorro. Por una parte, se estaría reduciendo la cantidad de residuos de terrenos a gestionar $\mathrm{y}$, por otra, se estaría minimizando la compra de tierras suministradas para rellenos o subbases. Ya se han desarrollado estudios similares verificando que, en función del porcentaje de tierras a reutilizar, que oscila entre $30 \%$ y $89 \%$ de reutilización, el porcentaje de ahorro fluctúa entre el 25\% y el $75 \%$ (RIVERO-CAMACHO; SOLÍS-GUZMÁN; MARRERO, 2015). Así mismo, si se diera la circunstancia de suelos con vegetación de árboles y/o arbustos, su venta como biomasa puede llegar a generar ingresos para el proyecto gracias a una correcta gestión de los RCD (MARRERO et al., 2017). En este último caso, si se clasifican los RCD del caso de estudio de acuerdo con las categorías presentadas en la Figura 8, se obtiene el gráfico presentado en la Figura 10.

Por su parte, en la Figura 11 se puede observar de forma más gráfica las proporciones de RCD en proyectos de urbanización, a partir de una comparativa del caso chileno estudiado con uno español de proporciones similares. Es posible comprobar que las proporciones son semejantes en ambos casos, así como la viabilidad de la adaptación del modelo de cuantificación. De las diferencias que se pueden observar en dicha comparación, se destaca un mayor volumen de tierras en el caso español, debido a las mayores irregularidades del terreno estudiado, así como a las plantas de sótanos presentes en el proyecto. Por el contrario, en el caso chileno, se registra mayor volumen de residuos provenientes de los hormigones, debido a las demoliciones de los viales existentes en la parcela antes del comienzo de las obras.

Figura 9 - Proporción en volumen $\left(\mathrm{m}^{3}\right)$ de los RCD tras la separación

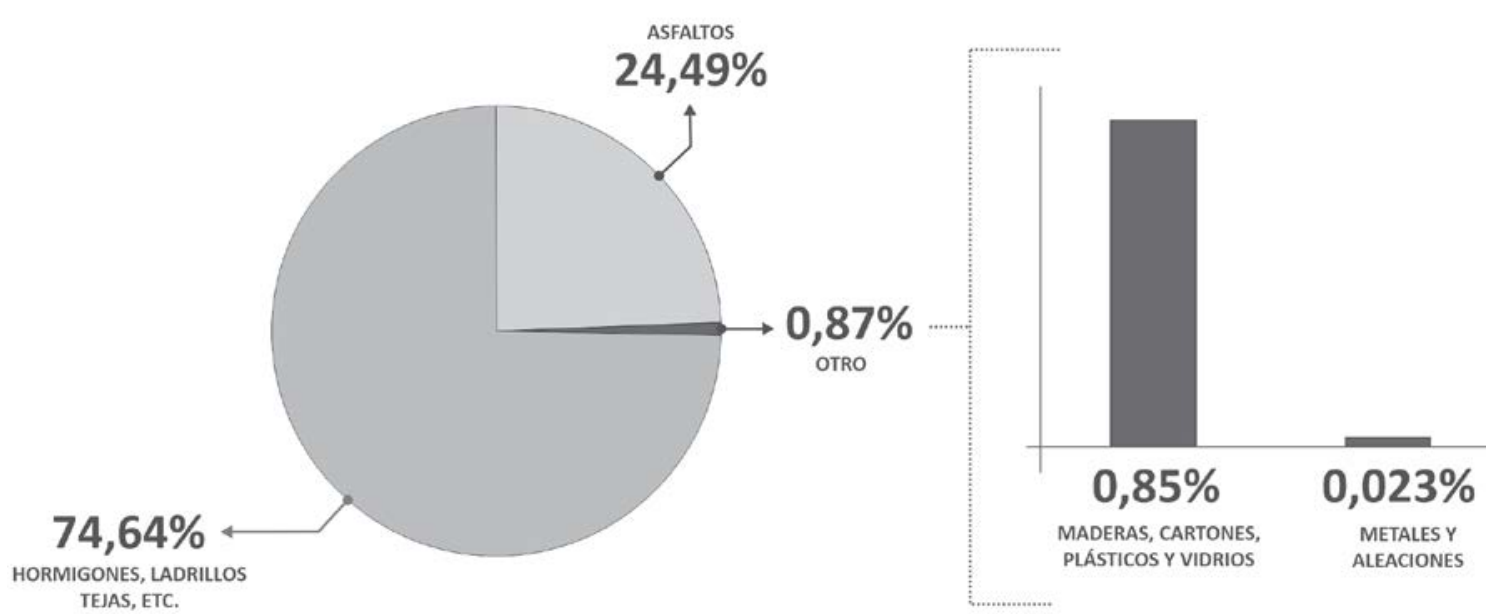


Figura 10 - Proporción en volumen $\left(\mathrm{m}^{3}\right)$ de los RCD tras la separación en cinco proyectos de urbanización españoles

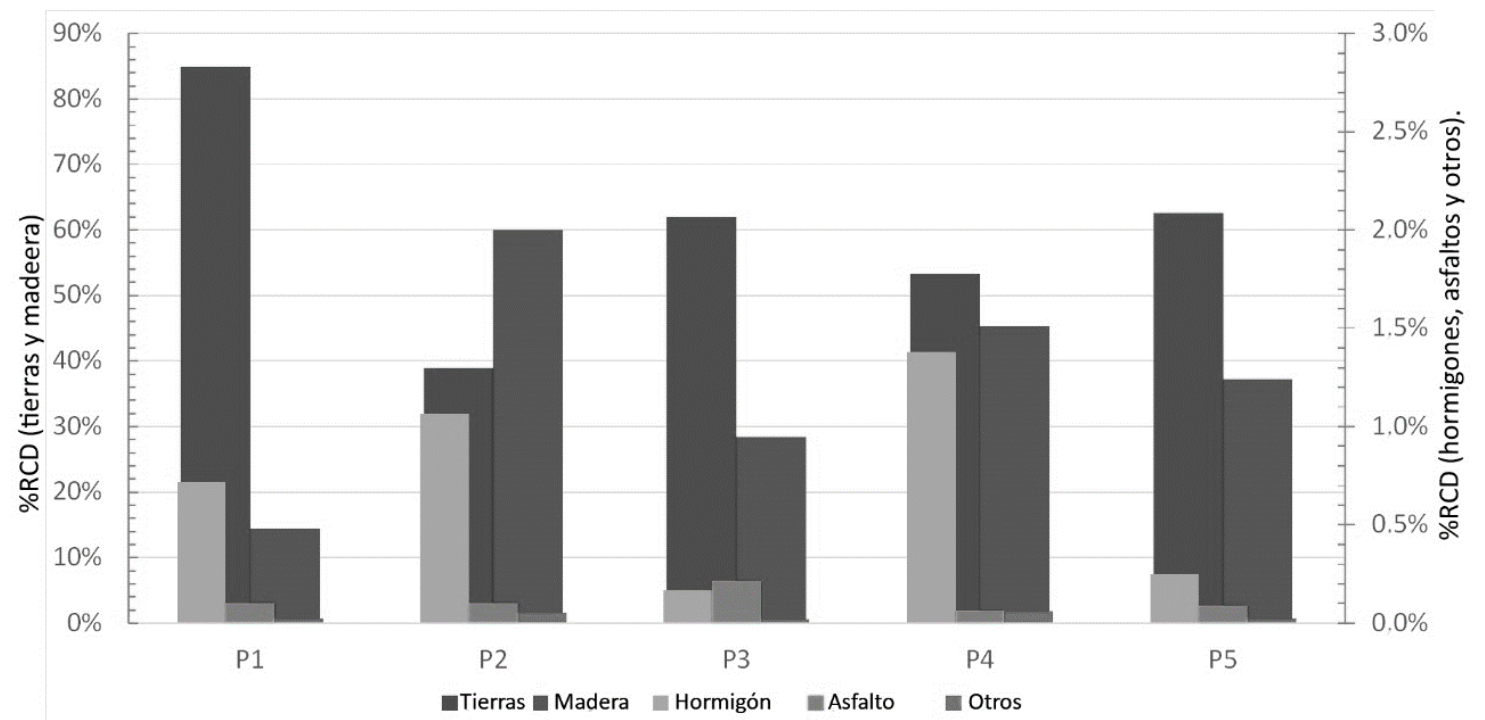

Fuente: Marrero et al. (2017).

Figura 11 - Comparativa del caso de estudio chileno con un caso similar español

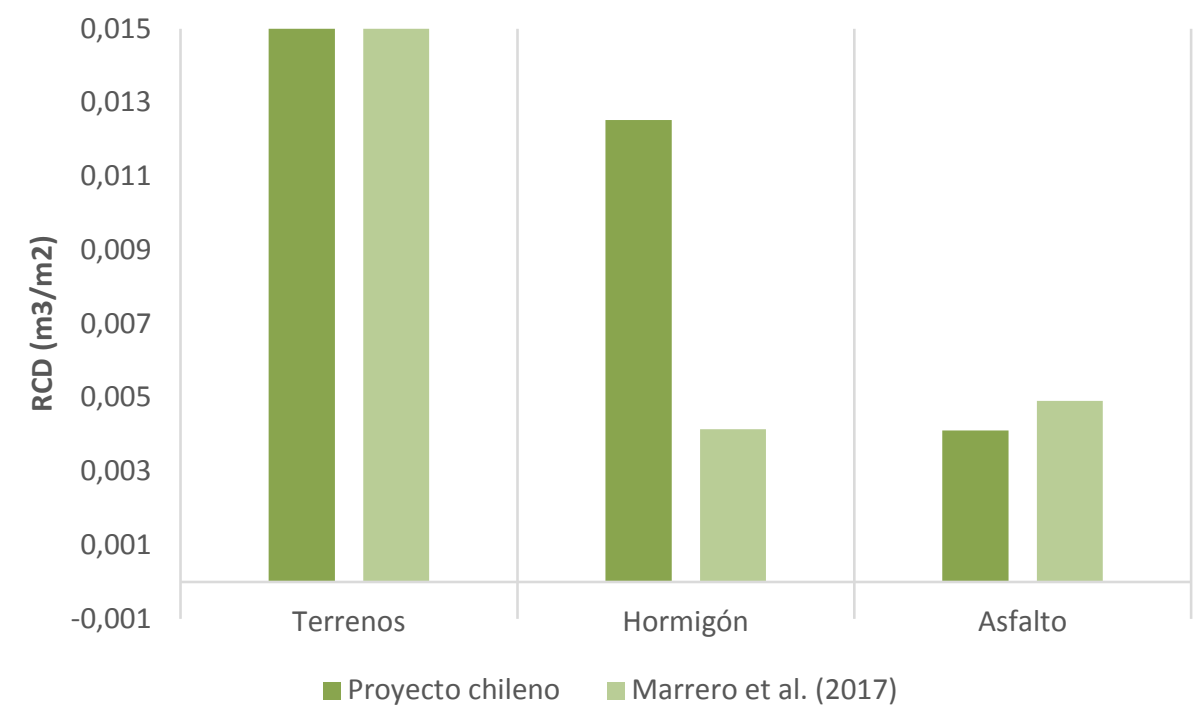

Si se comparan los resultados con los de otros autores, excluyendo las tierras, son solo un $10 \%$ de los recogidos en Brasil donde se tomaron datos en 20 obras (MACIEL; STUMPF; KERN, 2016), y la generación de RCD fue de $0,128 \mathrm{~m}^{3} / \mathrm{m}^{2}$ a 0,162 $\mathrm{m}^{3} / \mathrm{m}^{2}$ construido. Esta gran discrepancia se debe a que el presente caso de estudio se centra sólo en los trabajos de urbanización y excluye la completa construcción de los edificios, siendo estos últimos los resultados más comúnmente encontrados en la literatura (WU et al., 2014). Por otro lado, si se coteja la naturaleza de los residuos encontrados, por ejemplo, en el trabajo de Wang et al. (2018), en China, los principales RCD son también el hormigón (58,9\%), la cerámica (29,3\%) y los morteros $(9,8 \%)$.

\section{Comparativa de costos de gestión con la metodología chilena actual}

Para poder analizar mejor las ventajas de la metodología de separación de los RCD en obra, se calculará el costo que supondría la gestión de los RCD generados en el caso de estudio si se gestionaran en su totalidad como volumen de residuos mixtos. Para ello, será usado el precio de la BCCA establecido para cubas de RCD mixtos (Cuadro 6). 
Tomando el volumen total de RCD generados en el caso de estudio - 1.483,576 $\mathrm{m}^{3}$ (rever Cuadro 4) y multiplicándolo por el costo de gestión de un $\mathrm{m}^{3}$ de RCD mixtos (rever Cuadro 6), se obtiene un costo total para la gestión sin separación en obra de $€ 34.834,36$. Si se observa el total del presupuesto generado anteriormente (rever Cuadro 5), $€$ 15.435,31 se puede comprobar que, usando la metodología planteada con separación en obra, los costos de gestión del caso de estudio se reducen un $56 \%$.

En la actualidad, pocas son las empresas chilenas que proporcionan servicios de retirada selectiva de RCD en las obras. Por ahora, lo tradicional en Chile es retirar los RCD de forma conjunta, con un costo asociado de CLP\$ 50.000-55.000 por cada $\mathrm{m}^{3}$ de RCD mixto (Cuadro 7). Por ese motivo, la gestión de RCD del caso de estudio tendría un costo que rondaría los CLP\$ 74.178.800.

El hecho de que este costo pueda reducirse en torno del $50 \%$ es una razón de peso para plantearse el cambio de estrategia y empezar a implantar un nuevo sistema de gestión de RCD en países latinoamericanos como Chile. Es importante destacar que esto no sólo traerá beneficios a los mandantes de los proyectos, sino que reducirá en gran medida los impactos al medioambiente asociados a las obras de construcción.

\section{Cuadro 6 - Precio de gestión de RCD mixtos de acuerdo con la BCCA}

\begin{tabular}{|c|c|c|c|c|c|}
\hline 17RRR00220 & $\mathbf{m}^{3}$ & \multicolumn{4}{|c|}{$\begin{array}{l}\text { RETIRADA DE RESIDUOS MIXTOS N.P. A PLANTA DE } \\
\text { VALORIZ. } 15 \mathrm{~km} .\end{array}$} \\
\hline \multicolumn{6}{|c|}{$\begin{array}{l}\text { Retirada de residuos mixtos en obra de nueva planta a planta de valorización situada a una distancia } \\
\text { máxima de } 15 \mathrm{~km} \text {. Consiste en: transporte interior, carga, transporte a planta, descarga y canon de } \\
\text { gestión. }\end{array}$} \\
\hline \multicolumn{6}{|c|}{ Medido el volumen esponjado } \\
\hline Código & & Concepto & Cantidad & $\begin{array}{c}\text { Precio unitario } \\
(€)\end{array}$ & Importe (€) \\
\hline AER00100 & $\begin{array}{l}\mathrm{m}^{3} \mathrm{~T} \\
\text { mecé } \\
\text { mixt }\end{array}$ & $\begin{array}{l}\text { nsporte interior } \\
\text { ico de residuos }\end{array}$ & 1,00 & 2,82 & 2,82 \\
\hline ER00100 & $\begin{array}{l}\mathrm{m}^{3} \mathrm{C} \\
\text { resid }\end{array}$ & $\begin{array}{l}\text { on de gestión de } \\
\text { os mixtos }\end{array}$ & 1,00 & 12,50 & 12,50 \\
\hline ME00300 & $\mathrm{hPa}$ & cargadora & 0,020 & 23,87 & 0,48 \\
\hline \multirow[t]{2}{*}{ MK00100 } & $\mathrm{h} \mathrm{Ca}$ & ión basculante & 0,300 & 25,60 & 7,68 \\
\hline & & & \multicolumn{2}{|c|}{ Costos directos } & $€ 23,48 / \mathrm{m}^{3}$ \\
\hline
\end{tabular}

Cuadro 7 - Precio de gestión de RCD mixtos en Chile

\begin{tabular}{|c|c|c|c|c|}
\hline 17RRR10220 & \multicolumn{4}{|c|}{$\begin{array}{l}\text { RETIRADA DE RESIDUOS MIXTOS N.P. A PLANTA } \\
\text { DE VALORIZ. } 15 \mathrm{~km} .\end{array}$} \\
\hline \multicolumn{5}{|c|}{$\begin{array}{l}\text { Retirada de residuos mixtos en obra de nueva planta a planta de valorización situada a una } \\
\text { distancia máxima de } 15 \mathrm{~km} \text {. Consiste en: transporte interior, carga, transporte a planta, } \\
\text { descarga y canon de gestión. }\end{array}$} \\
\hline \multicolumn{5}{|c|}{ Medido el volumen esponjado } \\
\hline Código & Concepto & Cantidad & $\begin{array}{l}\text { Precio unitario } \\
\text { (CLP\$) }\end{array}$ & Importe (CLP\$) \\
\hline AER00100 & $\begin{array}{l}\mathrm{m}^{3} \text { Transporte } \\
\text { interior mecánico de } \\
\text { residuos mixtos }\end{array}$ & 1,00 & $6.005,11$ & $6.005,11$ \\
\hline ER00100 & $\begin{array}{l}\mathrm{m}^{3} \text { Canon de } \\
\text { gestión de residuos } \\
\text { mixtos }\end{array}$ & 1,00 & $26.618,40$ & $26.618,40$ \\
\hline ME00300 & h Pala cargadora & 0,020 & $51.107,5$ & $1.022,15$ \\
\hline \multirow[t]{2}{*}{ MK00100 } & $\begin{array}{l}\text { h Camión } \\
\text { basculante }\end{array}$ & 0,300 & $54.514,47$ & $16.354,34$ \\
\hline & & \multicolumn{2}{|c|}{ Costos directos } & CLP\$ 50.000/ m3 \\
\hline
\end{tabular}




\section{Conclusiones}

La aplicación de esta metodología en la industria de la construcción chilena se traduce en un triple beneficio: un beneficio ambiental directo, un beneficio industrial, dado que genera nuevas oportunidades de negocio a partir de la gestión ecoeficiente de RCD, y un evidente impacto en la dimensión social. Igualmente, se puede ver un horizonte de mejores perspectivas para Chile, con mejora de la calidad de vida y aumento de la competitividad del sector, así como generación de empleos más seguros y productivos, mayor provisión de servicios públicos con los mismos recursos, ahorros operacionales en edificación pública, calidad y eficiencia del stock edificado y mejora de la calidad ambiental de edificios y ciudades chilenas.

La aplicación de la metodología española a un proyecto de urbanización chileno permitió comprobar que es viable su adaptación para estimar las cantidades de RCD generadas en las obras de construcción realizadas en el país.

Considerando el escenario chileno, para que el modelo estudiado tenga éxito y sea efectivo, debe estar acompañado de gobernanza y legislación adecuadas que lo sustente y que obligue, entre otras cuestiones, a definir las cantidades de RCD esperadas en las obras y su eventual reutilización, reciclaje, tratamiento o eliminación controlada.

La gestión de RCD a partir de la separación de residuos en obra según su naturaleza - y en la obra analizada en este artículo en particular, debido al gran volumen de tierras y hormigones que pueden ser fácilmente reutilizados y reciclados - permite que el potencial de ahorro llegue a más del $50 \%$. Sin embargo, en obras en las que no es posible reutilizar todas las tierras y/o hormigones, el ahorro puede ser significativamente menor, de alrededor de $25 \%$

El alto porcentaje de tierras en la totalidad de los residuos generados en las obras de urbanización las torna un elemento clave para optimizar los gastos en la gestión de urbanización.

Entre las mayores barreras para la implementación del modelo español en Chile, se identifica la falta de control administrativo directo, que puede llevarse a cabo por medio del visado de proyectos por parte de los municipios, así como en España. También se identifica como factor muy importante para permitir la implantación de los modelos de control el facilitar, por medio de bancos de costos de la construcción abiertos y públicos, la estimación de las cantidades de RCD y su presupuesto.

Todo lo anterior debe estar vinculado, en primer lugar, al presupuesto de ejecución material del proyecto y, en segundo lugar, a que se pueda trabajar en la reducción de estos costos. Para ello, es necesario vincular la cuantificación al presupuesto, ya que gestionar bien los RCD no es solo medioambientalmente sostenible, sino que también es económicamente viable.

\section{Referencias}

AGUIRRE, C. et al. Diagnóstico de la Generación de Residuos Sólidos de Construcción en Obras de Edificación en Altura en la Región Metropolitana. Revista de la Construcción, v. 4, n. 2, p. 38-46, 2005.

BELTRÁN RIAÑO, J. W. Análisis de Alternativas Para la Gestión Ambiental de los Residuos de Demolición y Construcción (RCD), en la Ciudad de Bogotá a Partir del Ciclo de Vida y la Economía Circular: planeación ambiental y manejo integral de los recursos naturales. Universidad Militar Nueva Granada, 2017.

BRITO, M. Construcción Limpia: impactos y desafíos de la gestión de residuos. In: CONSTRUYE 2025: Seminario Construcción Limpia: Impactos y Desafíos de la Gestión de Residuos, 12 de abril de 2018. Disponible en: $<$ http://construye2025.cl/seminario-construccionlimpia-impactos-y-desafios-de-la-gestion-deresiduos/>. Acceso en: 03 abr. 2019.

CÁMARA CHILENA DE LA CONSTRUCCIÓN. Informe MACh: Macroeconomía y construcción. 2018.

CHENG, J. C.; MA, L. Y. A BIM-Based System for Demolition and Renovation Waste Estimation and Planning. Waste Management, v. 33, n. 6, p. 1539-1551, jun. 2013.

COCHRAN, K. M.; TOWNSEND, T. G. Estimating Construction and Demolition Debris Generation Using a Materials Flow Analysis Approach. Waste Management, v. 30, n. 11, p. 2247-2254, 2010.

COELHO, A.; BRITO, J. de. Generation of Construction and Demolition Waste in Portugal. Waste Management \& Research, v. 29, n. 7, p. 739-750, 2011.

COMUNIDAD AUTÓNOMA DEL BCCA. Base de Costes de la Construcción de Andalucía (BCCA), 19 julio 2017. Banco de precios. Andalucía: Consejería de Fomento y Vivienda (CFV), Universidad de Sevilla y Colegio Oficial de Aparejadores y Arquitectos Técnicos de Sevilla, 2017. 
DEL RÍO, M.; VILLORIA, P.; TORRIJOS, F. Reverse Logistics Applied to Building Companies. Demolition Stage $=$ Logística Inversa aplicada a las Empresas de Edificación: fase de demolición. Building \& Management, v. 1, n. 2, p. 12-23, 2017.

ESPAÑA. Ministerio de la Presidencia. Real Decreto 105/2008, de 1 de febrero, por el que se regula la producción y gestión de los residuos de construcción y demolición. Diario Oficial del Estado, n. 38. España, 2008. Disponible en: $<$ https://www.boe.es/buscar/pdf/2008/BOE-A2008-2486-consolidado.pdf $>$. Acceso en: 03 abr. 2019.

ESPAÑA. Ministerio de Medio Ambiente. Orden MAM/304/2002, de 8 de febrero, por la que se publican las operaciones de valorización y eliminación de residuos y la lista europea de residuos. Diario Oficial del Estado, n. 43. España, 2002. Disponible en: <https://www.boe.es/boe/dias/2002/02/19/pdfs/A0 6494-06515.pdf>. Acceso en: 03 abr. 2019.

\section{EUROSTAT. Recovery Rate of Construction} and Demolition Waste. 2018. Disponible en: $<$ https://ec.europa.eu/eurostat/tgm/table.do?tab=ta ble\&init $=1 \&$ language $=$ en $\&$ pcode $=$ cei_wm040\&pl ugin=1>. Acceso en: 03 abr. 2019.

FORMOSO, C. T. et al. The Identification and Analysis of Making-Do Waste: insights from two Brazilian construction sites. Ambiente

Construído, Porto Alegre, v. 17, n. 3, p. 183-197, jul./set 2017.

HORRACH, J. Otros Modelos de Gestión de Residuos: San Francisco, U.S.A, 17 de enero de 2017. Iresiduo, 2017. Disponible en: <http://iresiduo.com/blogs/juan-mateohorrach/otros-modelos-gestion-residuos-sanfrancisco-usa>. Acceso en: 03 abr. 2019.

INSTITUTO NACIONAL DE NORMALIZACIÓN. Ley 20.920: marco legal para la gestión de residuos, responsabilidad extendida del productor y fomento al reciclaje. Chile, 2016.

JEFATURA DEL ESTADO. Ley 22/2011, mod 12 de mayo de 2016. Ley 22/2011, de 28 de julio, de residuos y suelos contaminados. Diario Oficial del Estado, 2016. Disponible en: https://www.boe.es/eli/es///2011/07/28/22/con. Acceso en: 26 abr. 2019.

KOFOWOROLA, O.; GHEEWALA, S. H. Estimation of Construction waste Generation and Management in Thailand. Waste Management, v. 29, n. 2, p. 731-738, 2009.
KOURMPANIS, B. et al. Preliminary Study for the Management of Construction and Demolition Waste. Waste Management \& Research, v. 26, n. 3, p. 267-75, 2008.

LI, Y. et al. Developing a Quantitative Construction Waste Estimation Model for Building Construction Projects. Resources, Conservation and Recycling, v. 106, p. 9-20, 2016.

LLATAS, C. A Model for Quantifying Construction Waste in Projects According to the European Waste List. Waste Management, v. 31, n. 6, p. 1261-1276, 2011.

MACIEL, T.; STUMPF, M.; KERN, A. Propuesta de Sistema de Gestión Para la Planificación y Control de Residuos de la Construcción. Revista Ingeniería de Construcción, v. 31, n. 2, 2016.

MARRERO, M. et al. Assessing the Economic Impact and Ecological Footprint of Construction and Demolition Waste During the Urbanization of Rural Land. Resources, Conservation and Recycling, v. 117, part B, p. 60-174, 2017.

MARRERO, M. et al. Demolition Waste Management in Spanish Legislation. The Open Construction and Building Technology Journal, v. 5, p. 162-173, 2011.

MARRERO, M.; RAMIREZ-DE-ARELLANO, A. The Building Cost System in Andalusia: application to construction and demolition waste management. Construction Management and Economics, v. 28, n. 5, p. 495-507, 2010.

MCBEAN, E. A.; FORTIN, M. H. P. A Forecast Model of Refuse Tonnage with Recapture and Uncertainty Bounds. Waste Management \& Research, v. 11, n.5, p. 373-385, 1993.

MERCADER-MOYANO, P.; RAMIREZ-DEARELLANO, A. Selective Classification and Quantification Model of C\&D Waste from Material Resources Consumed in residential Building Construction. Waste Management \& Research, v. 31, n. 5 p. 458-474, 2013.

MOCHE SCHNEIDER, D.; PHILIPPI JUNIOR, A. Gestão Pública de Resíduos da Construçao Civil no Município de São Paulo. Ambiente Construído, Porto Alegre, v. 4, n. 4, p. 21-32, out./dez. 2004.

MOKHTAR, S. N. et al. Factors That Contribute to the Generation of Construction Waste at Sites. Advanced Materials Research, v. 163-167, p. 4501-4507, 2011. 
MORAGA, E. El Giro Sustentable del Sector Inmobiliario. La Tercera, Pulso, 2018. Disponible en: <https:/www.latercera.com/pulso/noticia/girosustentable-del-sector-inmobiliario/166704/> . Acceso en: 03 abr. 2019.

MORÁN DEL POZO, J. M. et al. Estado Actual de la Gestión de Residuos de Construcción y Demolición: limitaciones. State of the art on construction and demolition wastes management: limitations. Informes de la Construcción, v. 63, n. 521, p. 89-95, 2011.

OEKO-SERVICE LUXEMBOURG. Super Drecks Kescht fir Betriber, 2002. Disponible en: $<$ http://www.superdreckskescht.lu>. Acceso en: 03 abr. 2019.

PÉREZ-CARMONA, J. J.; MARRERO, M.; SOLÍS-GUZMÁN, J. Programa Informático: modelo de cuantificación de RCD ajustado al real decreto 105/2008. Modelo de utilidad, propiedad industrial. Solicitud: 2013-03-07. Universidad de Sevilla, 2013.

\section{PUERTO, M. Cuantificación de Residuos de} Construcción y Demolición en la Transformación de Terrenos Rústicos en Urbanos. Fase de urbanización. TFM Máster gestión integral en edificación. Universidad de Sevilla, 2011.

RAMÍREZ-DE-ARELLANO, A. et al. Retirada Selectiva de Residuos: modelo de presupuestación. Fundación Cultural del Colegio Oficial de Aparejadores y Arquitectos Técnicos de Sevilla, v. 6, p. 204, 2002.

RIVERO-CAMACHO, C.; SOLÍS- GUZMÁN, J.; MARRERO, M. Gestión y Aprovechamiento de Residuos en la Urbanización del Suelo. In: CONGRESO INTERNACIONAL DE CONSTRUCCIÓN SOSTENIBLE Y SOLUCIONES ECO-EFICIENTES, 2., Sevilla, 2015. Proceedings... Sevilha: Universidad de Sevilla, 2015.

SERRA NAVARRETE, A. Gestión de la Prevención de Riesgos Laborales y Gestión de Residuos de Demolición en el Sector de la Construcción: comparación de normativa de España con la de Chile. Tesis - Máster Universitario en Planificación y Gestión en Ingeniería Civil, Universitat Politècnica de València. Canales y Puertos, 2014.

SOLÍS-GUZMAN, J. et al. A Spanish Model for Quantification and Management of Construction Waste. Waste Management, v. 29, n. 9, p. 25422548, 2009.
SOLÍS-GUZMAN, J.; MARRERO, M.; GUISADO GARCÍA, D. Model for the Quantification and Budgeting of the Construction and Demolition Waste: application to roads. Carreteras: Revista técnica de la Asociación Española de la Carretera, v. 4, n. 195, p. 6-18, 2014.

SOUSA, D. S. V. de; CÂNDIDO, L. F.; BARROS, N. J. de P. Medição de Desempenho na Construção Civil: um estudo exploratório com construtoras cearenses. Ambiente Construído, Porto Alegre, v. 18, n. 1, p. 9-29, jan./mar. 2018.

SOUZA, U. E. L. et al. Diagnóstico e Combate à Geração de Resíduos na Produção de Obras de Construção de Edifícios: uma abordagem progressiva. Ambiente Construído, Porto Alegre, v. 4, n. 4, p. 33-46, out./dez. 2004.

SUÁREZ-SILGADO, S. et al. Diagnóstico y Propuestas Para la Gestión de los Residuos de Construcción y Demolición en la Ciudad de Ibagué (Colombia). Universidad Nacional de Colombia. Gestión y Ambiente, v. 21, n. 1, p. 9-21, 2018.

UNIÓN EUROPEA. Directiva 2008/98 / CE del Parlamento Europeo y del Consejo, de 19 de noviembre de 2008, sobre residuos y por la que se derogan determinadas directivas. Diario Oficial de la Unión Europea, 2008.

UNIVERSIDAD DEL BIO-BIO. EcoBarrios de

Madera: la apuesta del Citec UBB por una construcción sustentable. Dirección General de Investigación (DGI), 2018. Disponible en: <http://www.dgi.ubiobio.cl/dgi/index.php/2018/04 /05/ecobarrios-de-madera-la-apuesta-del-citecubb-por-una-construccionsustentable/?print=print>. Acceso en: 03 abr. 2019.

VILLORIA-SÁEZ, P.; PORRAS-AMORES, C.; DEL RÍO MERINO, M. New Quantification Proposal for Construction Waste Generation in New Residential Constructions. Journal of Cleaner Production, v. 102, p. 58-65, 2015.

WANG, J. et al. Critical Success Factors For onSite Sorting of Construction Waste: a china study resources. Resources Conservation and Recycling, v. 54, n. 11, p. 931-936, 2010.

WANG, T. et al. Estimating the Environmental Costs and Benefits of Demolition Waste Using Life Cycle Assessment and Willingness-to-Pay: a case study in Shenzhen. Journal of Cleaner Production, v. 1722, p. 14-26, 2018. 
WIMALASENA, B. A. D. S.; RUWANPURA, J. Y.; HETTIARATCHI, J. P. Modeling Construction Waste Generation Towards Sustainability. In: CONSTRUCTION Research Congress 2010: Innovation for Reshaping Construction Practice, 2010.

WU, Z. et al. Quantifying Construction and Demolition Waste: an analytical review. Waste Management, v. 34, n. 9, p. 1683-1692, 2014.

YOST, P. A.; HALSTEAD, J. M. A Methodology for Quantifying the Volume of Construction Waste. Waste Management \& Research, v. 14, n. 5, p. 453-461, 1996.
YUAN, H. et al. A Model for Cost-Benefit Analysis of Construction and Demolition Waste Management Throughout the Waste Chain.

Resources, Conservation and Recycling, v. 55, n. 6, p. 604-612, 2011.

\section{Agradecimientos}

Este proyecto de investigación se desarrolló en el marco del trabajo colaborativo entre el Grupo de Investigación Arquitectura y Construcción Sustentable adscrito a los Centros de Investigación en Tecnologías de la Construcción (CITEC-UBB Chile), Centro Intersdisciplinario para la Productividad y Construcción sustentable (CIPYCS -NODO SUR) y el Grupo de Investigación ARDITEC de la Universidad de Sevilla, España. Se agradece la colaboración de los investigadores participantes de ambos grupos.

\section{Claudia Marcela Muñoz Sanguinetti}

Departamento Ciencias de la Construcción | Universidad del Bío-Bío | Av. Collao, 1202 | Concepción-Chile | 4030000 | E-mail: clmunoz@ubiobio.cl

\section{Cristina Rivero Camacho}

Departamento Construcciones Arquitectónicas 2 | Universidad de Sevilla | Avd. Reina Mercedes, 4 | Sevilla - España | 41012 | Tel.: +(034) 95455-6697 | E-mail: crivero1@us.es

\section{Madelyn Marrero Meléndez}

Departamento Construcciones Arquitectónicas 2 | Universidad de Sevilla | E-mail: madelyn@us.es

\section{Gabriel Cereceda Balic}

Departamento de Arte y Teoría del Diseño | Universidad del Bío-Bío | E-mail: cereceda@ubiobio.cl

Revista Ambiente Construído

Associação Nacional de Tecnologia do Ambiente Construído

Av. Osvaldo Aranha, 99 - 3o andar, Centro

Porto Alegre - RS - Brasil CEP $90035-190$

Telefone: +55 (51) 3308-4084

Fax: +55 (51) 3308-4054

www. seer. ufrgs. br/ ambienteconstruido

E-mail: ambienteconstruido@ufrgs.br 